\title{
66 \\ DYNAMIC BEHAVIOUR OF COUPLED SOIL-STRUCTURE SYSTEMS BY MEANS OF FEM ANALYSIS FOR THE SEISMIC RISK MITIGATION OF INGV BUILDING IN CATANIA (ITALY),
}

\author{
Maria Rosella Massimino ${ }^{1}$, Glenda Abate $^{1, *}$, Sebastiano Corsico ${ }^{1}$, Salvatore Grasso ${ }^{1}$, \\ Ernesto Motta ${ }^{1}$
}

${ }^{1}$ Dipartimento di Ingegneria Civile e Architettura, DICAR, Università degli Studi di Catania, Catania, Italy

Article history

Receveid March 6, 2017; accepted March 20, 2017.

Subject classification:

Full-coupled systems; Static and dynamic geotechnical characterization; Soil nonlinearity; Accelerations; Response and Fourier spectra.

\begin{abstract}
Seismic design of new structures, as well as retrofitting and/or improving of existing ones should be definitely considered a multidisciplinary subject, which depends on many factors, such as: local site effects and the dynamic interaction between the foundation soil and the structure. The accurate investigation on the structure and the surrounding soil is the first fundamental step for a realistic evaluation of the structure seismic performance. The present paper deals with the Dynamic Soil Structure Interaction (DSSI) analysis concerning the INGV (National Institute of Geophysics and Volcanology) building in Catania, by means of a FEM 2D modeling. The building is a prestigious masonry structure situated in an area characterized by a high seismic hazard. Several accelerograms scaled at the same PHA, with reference to the estimated seismicity of Catania, have been adopted. Soil properties were carefully investigated by means of static and dynamic in-situ and laboratory tests. Many investigations were also performed on the structure. Equivalent linear visco-elastic constitutive models have been adopted both for the soil and the structure. For considering soil nonlinearity, degraded shear modula $(G)$ and increased soil damping ratios $(D)$ have been evaluated for all the involved soil layers, according to two different approaches. Firstly, soil nonlinearity has been modeled basing on the EC8 [2003] suggestions; secondly, it has been modeled choosing the values of $G$ and $D$ according to the effective strain levels obtained for each soil layer and for each different input, by means of an iterative sub-routine. The dynamic response of the system has been analyzed in the time and frequency domains. Results are presented in terms of: acceleration amplification factors, Fourier and response spectra, amplification functions and shear forces per floor. The main goals of the paper are: i) to investigate the acceleration profiles along the soil and the structure considering and not considering the DSSI; ii) to investigate the soil filtering effect in terms of predominant frequency considering and not considering the DSSI; iii) to compare the obtained results with the ones given by a simpler 1D free-field soil analysis; iv) to compare the soil amplification factors and the response spectra obtained by 1D and 2D models with that by the Italian technical code [NTC, 2008]; v) to highlight the influence of DSSI in the seismic response of the structure; vi) to evaluate the influence of different modeling of soil nonlinearity on the dynamic response of the soil and structure.
\end{abstract}

\section{INTRODUCTION}

In engineering practice, seismic design of new structures and/or seismic retrofitting of existing ones are generally performed using the design spectra given by technical regulations and thus the pseudo-static approach. Alternatively, the designers can use dynamic analysis; in this case, the seismic motion is represented in terms of ground acceleration time-histories. In this second case, the seismic motion may be made by using artificial, recorded and simulated accelerograms. In sophisticated and appropriated structure design/retrofitting approaches the design spectra and/or the ground motion acceleration time-histories are derived by previous freefield (FF) site response analyses. The latter approach definitely represents an important step forward [Capilleri et al., 2003, 2005; Ferraro et al., 2016, 2018; Grasso and Maugeri, 2014; Castelli et al., 2018a,b], taking into account the specific conditions of subsoil and its fundamental filtering effects in terms of PHA and predominant frequency. Nevertheless, dynamic response at the foundation level of a structure deviates from the FF site response, because of kinematic and inertial interaction [Gazetas, 1991; Massimino et al., 2015; Abate et al., 2017a, b; Karatzetzou et al., 2017] and in different cases dynamic soil-structure interaction (DSSI) could be detri- 
mental for the structures [Mylonakis et al., 2000; Pandey et al., 2012]. In order to evaluate the effective ground motion at the foundation level it is necessary to investigate Dynamic Soil-Structure Interaction (DSSI). Since the 1970 s, DSSI has been investigated by means of theoretical approaches [Veletsos et al., 1974; Gazetas, 1983; Gazetas et al., 1991; Chatterjee et al., 2008; Voyagaki et al., 2013; Renzi et al., 2013], numerical modeling [Martin et al., 2001; Gazetas et al., 2004; Gajan et al., 2005, 2008; Massimino, 2005; Maugeri et al., 2012; Calvi et al.; 2014; Abate et al., 2015] as well as field and laboratory tests àCombescure et al., 2000; Faccioli et al., 2001; Prasad et al., 2004; Kutter et al., 2006; Ueng et al., 2006; Bienen et al., 2007; Ugalde et al., 2007; Anastasopoulos et al., 2013]. Particularly, numerical modeling of full-coupled soil-structure systems represent the most precious approach, being the nearest to the actual configurations to be analyzed [Abate et al., 2016, 2017c]. The great disadvantage of full-coupled numerical modeling has been the great time and memory efforts required until a short time ago. Thanks to the recent tremendous technological progress, this disadvantage is shrinking more and more.

The present paper deals with the numerical modeling of a full-coupled soil-structure system, involving the headquarters of the Catania Section of the National In- strain level based on resonant column tests. The results of the full-coupled system analyses have been compared with those related to the FF site response in the time and frequency domains, in terms of soil amplification ratio, Fourier and response spectra, and amplification functions. The achieved soil amplification ratio and response spectra have been also compared with those given by the Italian technical regulation NTC [2008]. Finally, the maximum shear forces at each structure level have been compared with those given by the fixed-base structure configuration, traditionally used in engineering practice.

\section{THE STRUCTURE AND ITS SUBSOIL}

The INGV building is located in the historic center of Catania and it was built at the end of 1800 . The building is a masonry structure (Figure 1) whose bearing walls were built of lava stone; the foundations are enlargements of these walls, and they were embedded for a depth equal to $2.5 \mathrm{~m}$. The floors are in brick and concrete downloading on curbs in reinforced concrete resting on the walls. The red line in Figure 1a identifies the INGV building.

As for the soil, in 2010 the red boreholes reported in Figure 2a were executed and laboratory and in situ tests

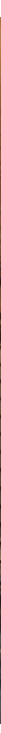

FIGURE 1. The INGV building; a) plan view; b) façade.

stitute of Geophysics and Volcanology (INGV). The building and its subsoil were subjected to investigations in the framework of the Research Project POR-FESR Sicilia 2007-2013, finalized to the reduction of the seismic risk in the Eastern Sicily. The seismic response of the full-coupled system has been investigated by means of a 2D FEM modeling, taking into account soil-nonlinearity according to EC8 [2003] as well as according to the reached were carried out. In 2014, the blue boreholes reported in Figure $2 \mathrm{~b}$ were executed. SPT, DH, CH and SDMT were also performed. Finally, laboratory tests for soil description and classification, direct shear tests, oedometer tests, resonant column tests and torsional shear tests were carried out on undisturbed samples. Then two cross-sections have been defined: the red and blue lines in Figure 3a. A third cross-section (green line) has been considered 
in the analyses, in order to take into account a section corresponding to the Northeast façade of the building (Figure3b). This cross-section has been made according to the red and blue cross-sections. Inclined layers have been considered, as shown in Figure 4. The bedrock is at more than $200 \mathrm{~m}$ from the ground level, but in the presented FEM analyses it has been fixed at $40 \mathrm{~m}$ (conventional bedrock), according to previous 1D analyses using

\section{THE FEM MODELING}

In order to evaluate the seismic response of the described soil-building system, a 2D finite element model has been developed by means of the ADINA code [ADINA, 2008]. The response of the system has been analyzed considering and not considering the DSSI, investigating different vertical alignments: along the

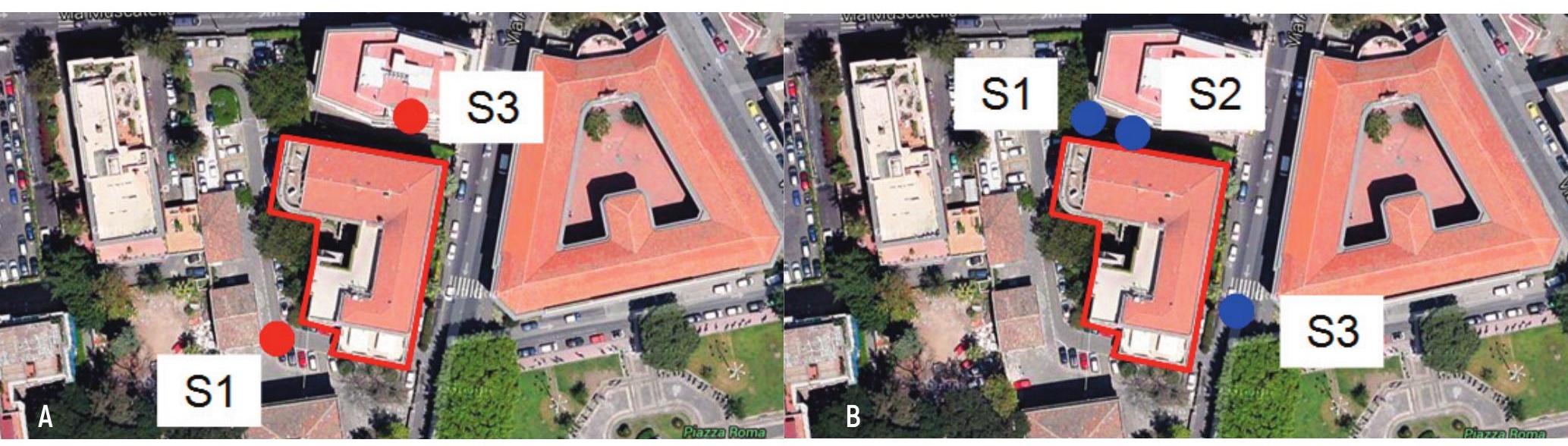

FIGURE 2. Boreholes during two different geotechnical investigations: a) 2010 campaign; b) 2014 campaign.
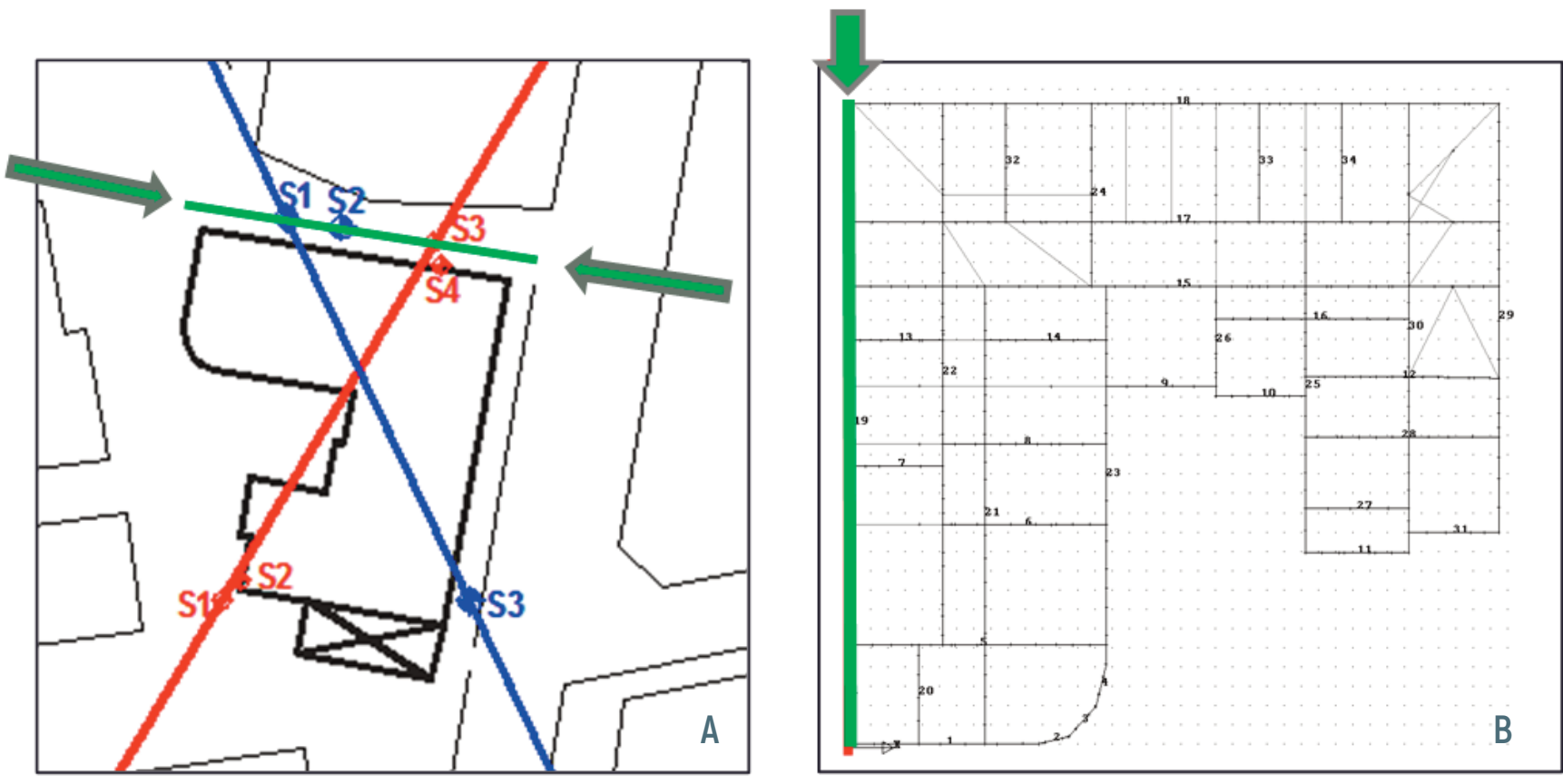

FIGURE 3. a) Plan view with boreholes and defined cross-sections; b) plan view of the structure with the analyzed section (see green line in Figure 3a).

STRATA code [Kottke et al., 2008], which showed no significant amplification from $\mathrm{z}=200 \mathrm{~m}$ to $\mathrm{z}=40 \mathrm{~m}$ (Figure $4 \mathrm{a})$. Figure $4 \mathrm{~b}$ shows the $V_{s}$ profiles; instead the main geotechnical properties are summarized in Table 1.

The adopted soil profile is shown in Figure 4c. The soil is of class C according to NTC [2008]. Figure 4c shows the soil profile along the investigated green line. structure (SSI) and far from the structure (FF). The results obtained by 2D FEM analysis have been also compared with those of simpler 1D analyses and with those of Italian technical code [NTC, 2008]. 3D analyses could be more realistic; nevertheless 3D analyses of the whole fullcoupled soil-structure system would result in a huge growth in the number of mesh nodes and elements, 


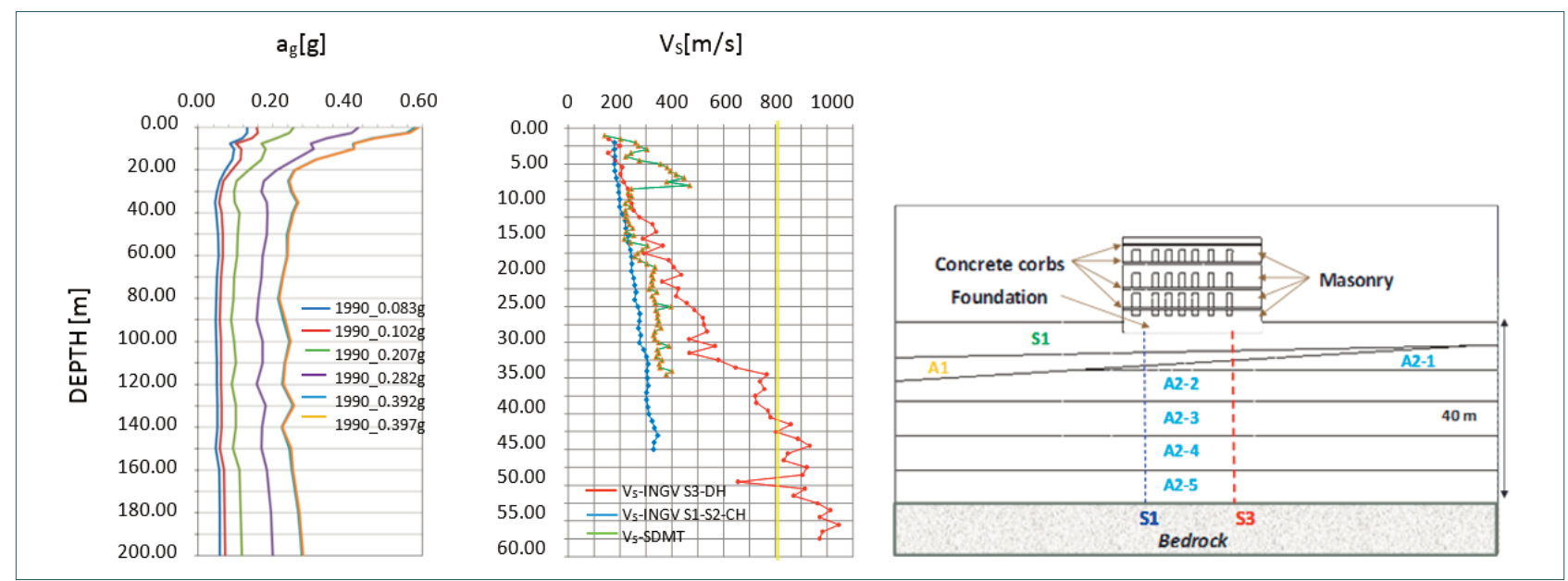

FIGURE 4. Adopted mesh with geometry, boundary and loading conditions.

\begin{tabular}{lcccccc} 
LAYER & $H(\mathrm{~m})$ & $V_{s}(\mathrm{~m} / \mathrm{s})$ & $v$ & $\rho\left(\mathrm{t} / \mathrm{m}^{3}\right)$ & $G_{0}(\mathrm{kPa})$ & $E_{0}(\mathrm{kPa})$ \\
\hline sandy silt (S1) & 6.7 & 179 & 0.25 & 2.04 & 65363 & 163409 \\
clay1 (A1) & 2.8 & 193 & 0.25 & 2.01 & 74870 & 187176 \\
clay2-1 (A2-1) & 1.6 & 197 & 0.25 & 2.01 & 78006 & 195015 \\
clay2-2 (A2-2) & 6.6 & 222 & 0.25 & 2.01 & 99061 & 247652 \\
clay2-3 (A2-3) & 7.2 & 253 & 0.25 & 2.01 & 128658 & 321645 \\
clay2-4 (A2-4) & 7.2 & 277 & 0.25 & 2.01 & 154225 & 385563 \\
clay2-5 (A2-5) & 7.2 & 310 & 0.25 & 2.01 & 193161 & 482903 \\
\hline
\end{tabular}

TABLE 1. Main geotechnical properties.

which in turn would lead to a vast increase in computing times. The results would be too dispersive and therefore unimportant for DSSI assessment. This is the reason why the authors have chosen to present in this paper the results of the 2D finite element model shown in Figure 5, using the same approach followed by many researchers
[Pitilakis et al., 2013; Anastasopoulos et al., 2014; Gazetas, 2015; Conti et al., 2017; Karatzetzou et al., 2017].

Figure 5 shows the adopted FEM mesh, including the geometry, boundary and loading conditions. The width of the soil deposit has been chosen in order to minimize as much as possible boundary effect; the height of the soil

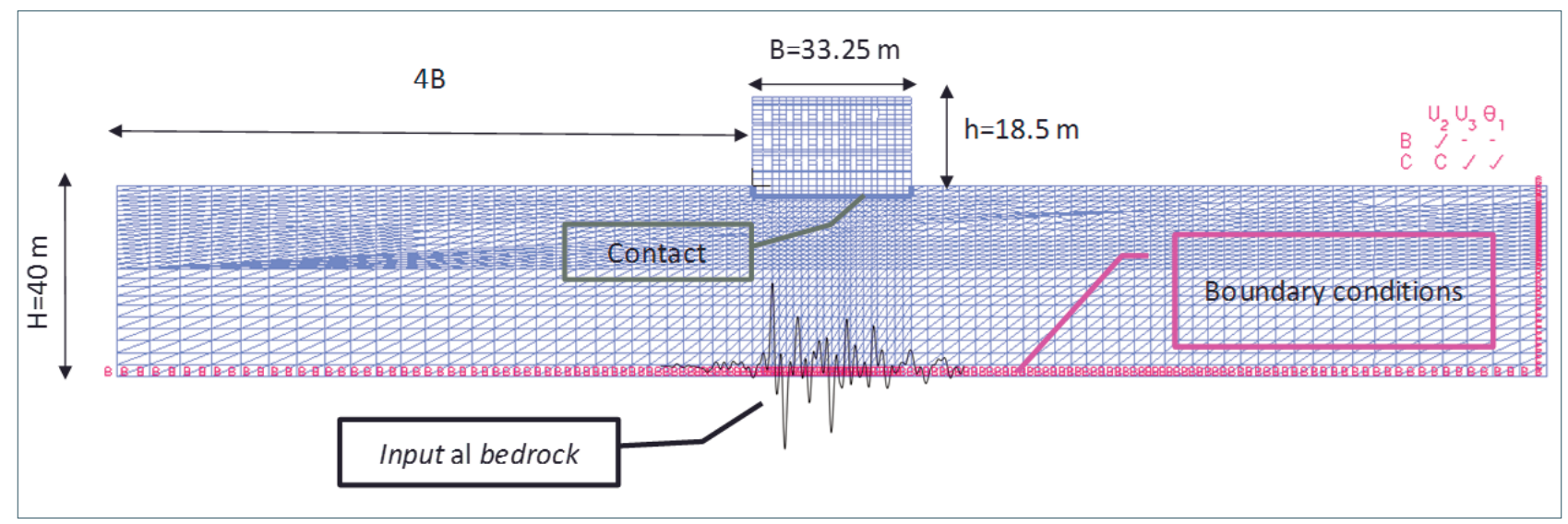

FIGURE 5. Adopted mesh with geometry, boundary and loading conditions. 


\begin{tabular}{|c|c|c|c|}
\hline ACCELERATION COEFFICIENT, $\alpha$ & DAMPING RATIO $D$ & $V_{s} / V_{s 0}$ & $G / G_{0}$ \\
\hline 0.10 & 0.03 & $0.9( \pm 0.07)$ & $0.80( \pm 0.10)$ \\
\hline 0.20 & 0.06 & $0.7( \pm 0.15)$ & $0.50( \pm 0.20)$ \\
\hline 0.30 & 0.10 & $0.6( \pm 0.15)$ & $0.36( \pm 0.20)$ \\
\hline
\end{tabular}

TABLE 2. Mean values ( \pm one standard deviation) of the damping coefficient $D$, of the shear waves $V_{s}$ and of the shear modulus $G$ according to EC8-5.

\begin{tabular}{|c|c|c|c|c|c|}
\hline LAYER & $H(\mathrm{~m})$ & $G_{\text {degr }}(\mathrm{kPa})$ & $D_{0, \text { incr }}(\%)$ & $E_{\text {degr }}(\mathrm{kPa})$ & $V_{s, \text { degr }}(\mathrm{m} / \mathrm{s})$ \\
\hline sandy silt (S1) & 3.4 & 28317 & 5.73 & 70793 & 118 \\
\hline clay1 (A1) & 8.2 & 15189 & 7.05 & 37972 & 87 \\
\hline clay2-1 (A2-1) & 10.1 & 22472 & 7.92 & 56180 & 106 \\
\hline clay2-2 (A2-2) & 14.0 & 19663 & 8.50 & 49158 & 99 \\
\hline clay2-3 (A2-3) & 21.2 & 14872 & 9.60 & 37181 & 86 \\
\hline clay2-4 (A2-4) & 28.7 & 13149 & 10.90 & 32873 & 81 \\
\hline clay2-5 (A2-5) & 36.2 & 11959 & 11.87 & 29898 & 77 \\
\hline
\end{tabular}

TABLE 3. Geotechnical properties adopted for the 7 different soil layers according to the effective strain level achieved for1693-1 seismic input.

\begin{tabular}{|c|c|c|c|c|c|}
\hline LAYER & $H(\mathrm{~m})$ & $G_{\text {degr }}(\mathrm{kPa})$ & $D_{0, \text { incr }}(\%)$ & $E_{\text {degr }}(\mathrm{kPa})$ & $V_{s, \text { degr }}(\mathrm{m} / \mathrm{s})$ \\
\hline sandy silt (S1) & 3.4 & 33277 & 5.40 & 83192 & 128 \\
\hline clay1 (A1) & 8.2 & 16540 & 6.84 & 41350 & 91 \\
\hline clay2-1 (A2-1) & 10.1 & 26688 & 7.32 & 66719 & 115 \\
\hline clay2-2 (A2-2) & 14.0 & 23910 & 7.70 & 59775 & 109 \\
\hline clay2-3 (A2-3) & 21.2 & 19763 & 8.40 & 49407 & 99 \\
\hline clay2-4 (A2-4) & 28.7 & 17428 & 8.90 & 43569 & 93 \\
\hline clay2-5 (A2-5) & 36.2 & 15485 & 9.34 & 38713 & 88 \\
\hline
\end{tabular}

TABLE 4. Geotechnical properties adopted for the 7 different soil layers according to the effective strain level achieved for $1693 \_2$ seismic input.

deposit derives from the previously discussed considerations about the conventional bedrock (See Section 2). The soil has been divided into 6 layers, some of which are inclined, according to the stratigraphy described in Figure 4c. About the boundary conditions, the nodes of the soil vertical boundaries have been linked by "con- straint equations" that imposed the same translation at the same depth [Abate et al., 2016]; all the nodes of the base of the mesh have been fixed in the vertical direction. Special contacts have been modelled between the building and the soil, in order to model possible foundation sliding and/or uplifting. 


\begin{tabular}{|c|c|c|c|c|c|}
\hline LAYER & $H(\mathrm{~m})$ & $G_{\text {degr }}(\mathrm{kPa})$ & $D_{0, \text { incr }}(\%)$ & $E_{\text {degr }}(\mathrm{kPa})$ & $V_{s, \text { degr }}(\mathrm{m} / \mathrm{s})$ \\
\hline sandy silt (S1) & 3.4 & 31815 & 5.50 & 79538 & 1245 \\
\hline clay1 (A1) & 8.2 & 16020 & 6.92 & 40050 & 89 \\
\hline clay2-1 (A2-1) & 10.1 & 27295 & 7.23 & 68238 & 117 \\
\hline clay2-2 (A2-2) & 14.0 & 24972 & 7.60 & 62430 & 111 \\
\hline clay2-3 (A2-3) & 21.2 & 20430 & 8.30 & 51075 & 101 \\
\hline clay2-4 (A2-4) & 28.7 & 18284 & 8.70 & 45709 & 95 \\
\hline clay2-5 (A2-5) & 36.2 & 16733 & 9.07 & 41833 & 91 \\
\hline
\end{tabular}

TABLE 5. Geotechnical properties adopted for the 7 different soil layers according to the effective strain level achieved for 1693_3 seismic input.

\begin{tabular}{|c|c|c|c|c|c|}
\hline LAYER & $H(\mathrm{~m})$ & $G_{\text {degr }}(\mathrm{kPa})$ & $D_{0, \text { incr }}(\%)$ & $E_{\text {degr }}(\mathrm{kPa})$ & $V_{s, \text { degr }}(\mathrm{m} / \mathrm{s})$ \\
\hline sandy silt (S1) & 3.4 & 18163 & 6.34 & 45407 & 94 \\
\hline clay1 (A1) & 8.2 & 17637 & 6.66 & 44093 & 94 \\
\hline clay2-1 (A2-1) & 10.1 & 40262 & 5.10 & 100656 & 141 \\
\hline clay2-2 (A2-2) & 14.0 & 41744 & 4.80 & 104360 & 144 \\
\hline clay2-3 (A2-3) & 21.2 & 40262 & 5.10 & 100656 & 141 \\
\hline clay2-4 (A2-4) & 28.7 & 38767 & 5.40 & 96917 & 139 \\
\hline clay2-5 (A2-5) & 36.2 & 37639 & 5.56 & 94098 & 137 \\
\hline
\end{tabular}

TABLE 6. Geotechnical properties adopted for the 7 different soil layers according to the effective strain level achieved for 1818 _1 seismic input.

\begin{tabular}{|c|c|c|c|c|c|}
\hline LAYER & $H(\mathrm{~m})$ & $G_{\text {degr }}(\mathrm{kPa})$ & $D_{0, \text { incr }}(\%)$ & $E_{\text {degr }}(\mathrm{kPa})$ & $V_{s, \operatorname{degr}}(\mathrm{m} / \mathrm{s})$ \\
\hline sandy silt (S1) & 3.4 & 44384 & 4.92 & 110961 & 148 \\
\hline clay1 (A1) & 8.2 & 24527 & 5.20 & 61316 & 110 \\
\hline clay2-1 (A2-1) & 10.1 & 41304 & 4.92 & 103260 & 143 \\
\hline clay2-2 (A2-2) & 14.0 & 38767 & 5.40 & 96917 & 139 \\
\hline clay2-3 (A2-3) & 21.2 & 35862 & 5.90 & 89656 & 134 \\
\hline clay2-4 (A2-4) & 28.7 & 34493 & 6.10 & 86232 & 131 \\
\hline clay2-5 (A2-5) & 36.2 & 32982 & 6.34 & 82456 & 128 \\
\hline
\end{tabular}

TABLE 7. Geotechnical properties adopted for the 7 different soil layers according to the effective strain level achieved for 1818_2 seismic input. 


\begin{tabular}{|c|c|c|c|c|c|}
\hline LAYER & $H(\mathrm{~m})$ & $G_{\text {degr }}(\mathrm{kPa})$ & $D_{0, \text { incr }}(\%)$ & $E_{\text {degr }}(\mathrm{kPa})$ & $V_{s, \text { degr }}(\mathrm{m} / \mathrm{s})$ \\
\hline sandy silt (S1) & 3.4 & 36785 & 5.17 & 91962 & 134 \\
\hline clay1 (A1) & 8.2 & 21764 & 6.00 & 54410 & 104 \\
\hline clay2-1 (A2-1) & 10.1 & 43803 & 4.48 & 109508 & 148 \\
\hline clay2-2 (A2-2) & 14.0 & 45155 & 4.20 & 112888 & 150 \\
\hline clay2-3 (A2-3) & 21.2 & 45155 & 4.20 & 112888 & 150 \\
\hline clay2-4 (A2-4) & 28.7 & 44683 & 4.30 & 112159 & 149 \\
\hline clay2-5 (A2-5) & 36.2 & 44447 & 4.37 & 111118 & 149 \\
\hline
\end{tabular}

TABLE 8. Geotechnical properties adopted for the 7 different soil layers according to the effective strain level achieved for 1818 _3 seismic input.

\begin{tabular}{lccccc} 
LAYER & $H(\mathrm{~m})$ & $G_{\text {degr }}(\mathrm{kPa})$ & $D_{0, \text { incr }}(\%)$ & $E_{\text {degr }}(\mathrm{kPa})$ & $V_{\mathrm{s}, \text { degr }}(\mathrm{m} / \mathrm{s})$ \\
sandy silt (S1) & 3.4 & 49623 & 4.88 & 124058 & 156 \\
clay1 (A1) & 8.2 & 18720 & 6.49 & 46799 & 97 \\
clay2-1 (A2-1) & 10.1 & 35832 & 5.87 & 89580 & 134 \\
clay2-2 (A2-2) & 14.0 & 34379 & 6.10 & 85947 & 131 \\
clay2-3 (A2-3) & 21.2 & 30602 & 6.70 & 76506 & 123 \\
clay2-4 (A2-4) & 28.7 & 28612 & 7.00 & 71529 & 119 \\
clay2-5 (A2-5) & 36.2 & 27521 & 7.20 & 68802 & 117 \\
\hline
\end{tabular}

TABLE 9. Geotechnical properties adopted for the 7 different soil layers according to the effective strain level achieved for1990 seismic input.

\begin{tabular}{lccc} 
& $E(\mathrm{kPa})$ & $v$ & $\mathrm{D}(\%)$ \\
\hline Masonry & 861 & 0.25 & 8 \\
Concrete Curb & 28757 & 0.3 & 5 \\
\hline
\end{tabular}

TABLE 10. Main structural properties.

Both the soil and the structure have been modelled by means of linear visco-elastic constitutive models. In particular, in this presented paper, a linear visco-elastic constitutive model has been chosen for the structure in order to focus attention on the effects of soil-nonlinearity, that is extremely important in the dynamic behaviour of full-coupled soil-structure systems [Abate et al., 2007; Pecker et al., 2010; Pecker et al., 2013; Mas- simino et al., 2015], even if this aspect is often neglected. Nevertheless, for the masonry, the value of $\mathrm{E}$ has been reduced to take into account the cracking of the sections, according to NTC [2008] and Ministerial Circular No. 617 of 2th February 2009, as it will explained deeply in the following.

As for the soil-nonlinearity, it has been considered as suggested by EC8 [2003] in a first step of analyses. As required by EC8 [2003] the coefficient $\alpha=S \times a_{g}(g)$, that is shown in the first column of Table 2 , is firstly computed. In the present case, it is $\alpha=1.29 \times 0.282=0.36$, being $S=1.29$ for soil type C according to NTC [2008] (see Section 2) and $a_{g}(g)=0.282$ (see the end of this section). Then, according to Table $2 G / G_{0}=0.36$ and $D=$ $10 \%$ have been fixed. In a second step of analyses, the values of $G$ and $D$ have been chosen according to the curves $G / G_{0}$ vs $\gamma$ and D vs $\gamma$ obtained by the performed Resonant Column Tests (RCT) shown in Figure 6, con- 


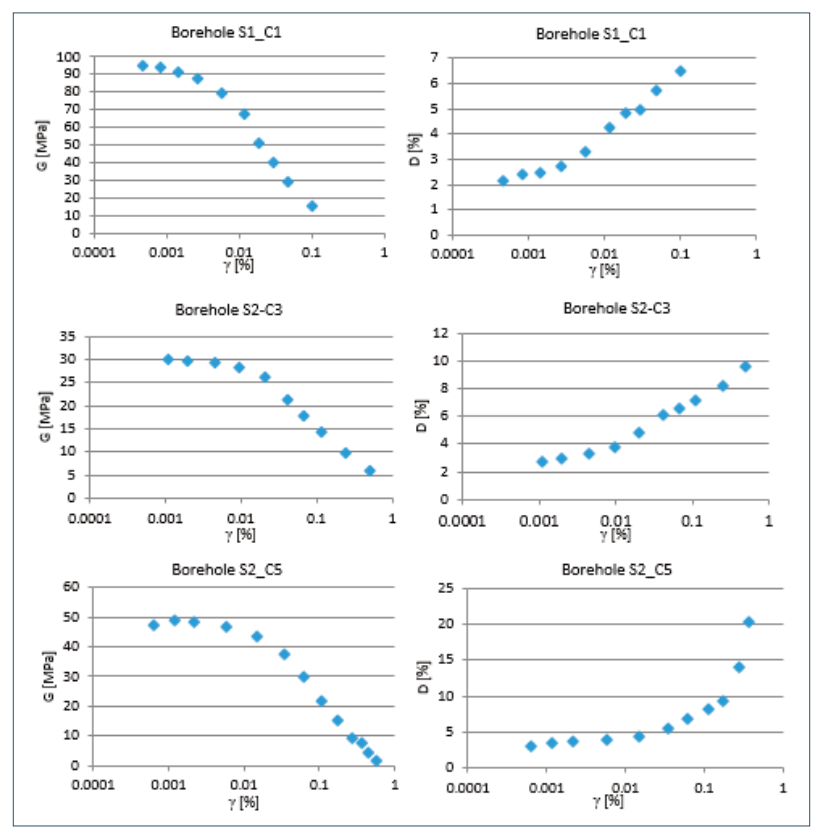

FIGURE 6. $G / G_{0}$ vs $\gamma$ and $D$ vs $\gamma$ curves, obtained by the performed Resonant Column Tests.

sidering the effective strain level $\gamma$ obtained for each soil layer and for each different input, according to an iter- ative sub-routine. Tables 3-9 shows the values achieved after the iterations, which are discussed in the following Section 4.4.

For the masonry, the value of $E$ has been reduced to take into account the cracking of the sections, according to Section 7.8.1.5.2 of NTC [2008] and Section C8A.2 of the Ministerial Circular No. 617 of 2th February 2009; typical values of $v$ and $D$ have been adopted [NTC, 2008]. For the concrete curbs, the value of $E$ has been adopted on the basis of tests carried out on the curbs, while the values of $v$ and $D$ have been adopted according to NTC [2008]. Table 10 shows the main structural properties.

The Rayleigh damping factors $\alpha$ and $\beta$ for the soil and the structure have been computed as $\alpha=D \cdot \omega$ and $\beta=$ $D / \omega$ [Lanzo et al., 2004], being $\omega$ and $D$ the natural frequency and the damping ratio of the soil or of the structure, respectively. As for the loading conditions, the computation of gravitational loads to be applied on the structure has been performed as suggested by NTC [2008]. The loads to be applied to the model were its weight and the loads distributed on the floors at various heights. The first ones have been imposed by applying to the entire model the "mass proportional load", such as the acceler-

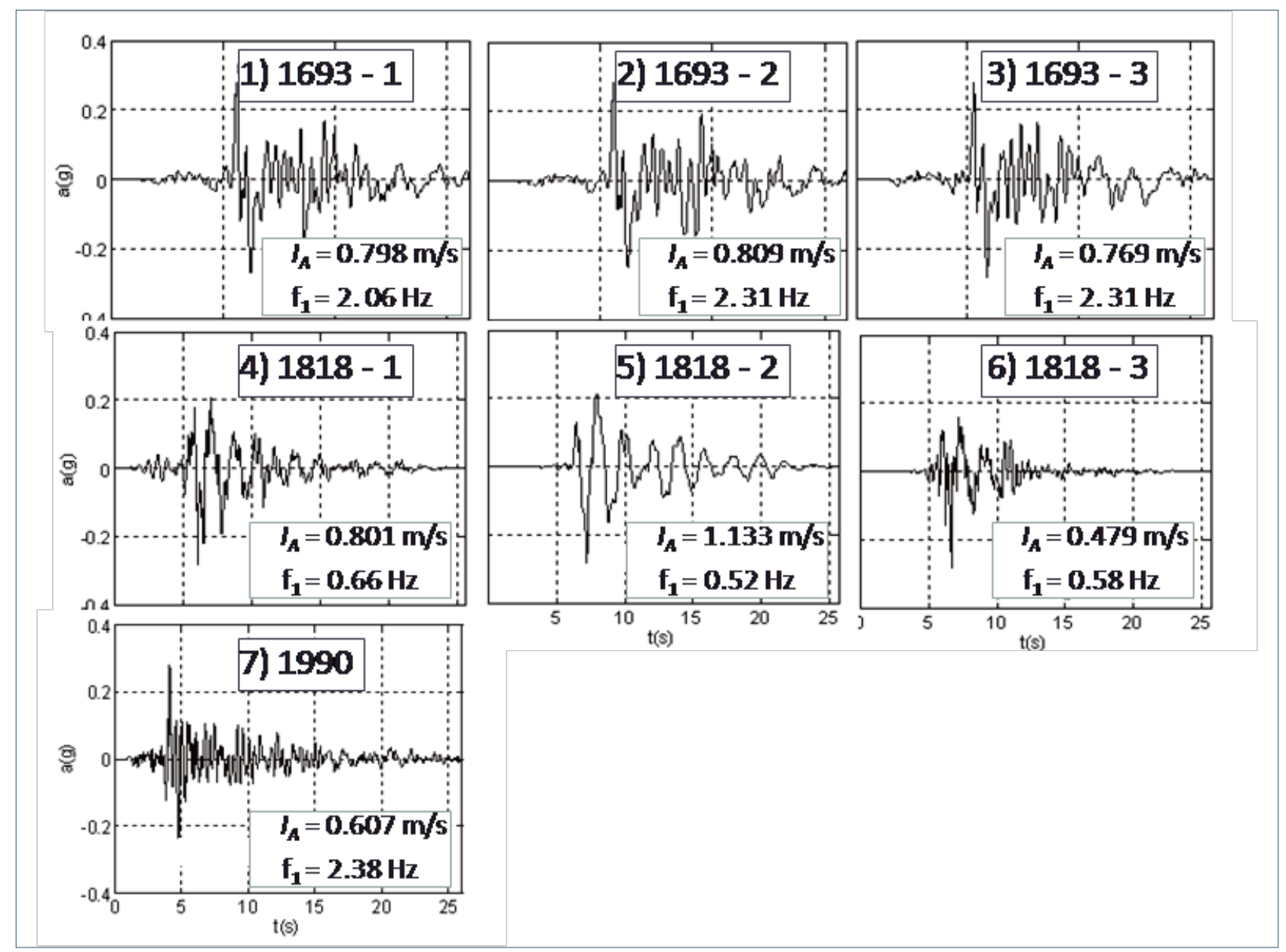

FIGURE 7. Adopted accelerogram time histories. 


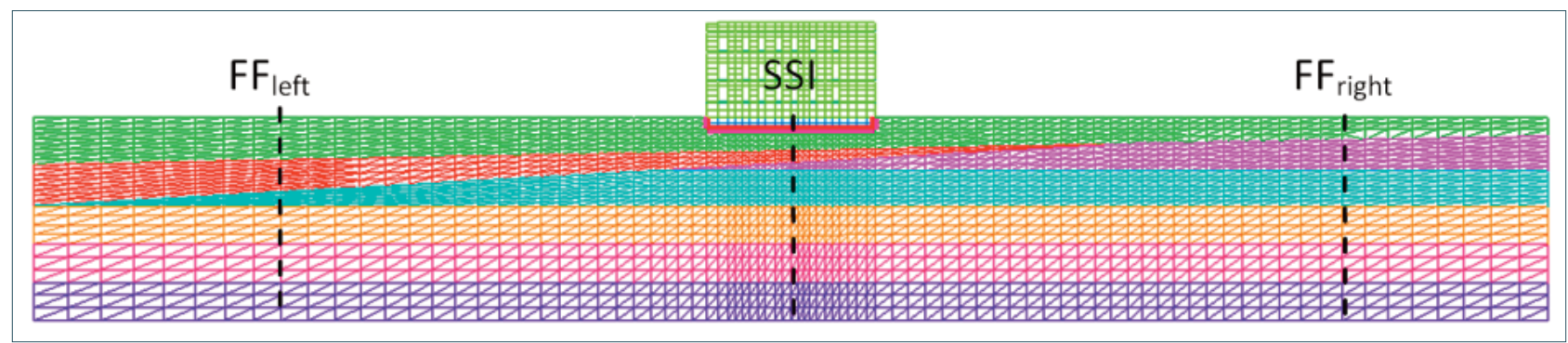

FIGURE 8. Location of the three investigated alignments (soil layer colours refer to the stratigraphy shown in Figure 4c).

ation of gravity in the vertical direction. The loads on the floors, through which the curbs weigh on the masonry, have been applied as concentrated masses on the curbs. So, the weight per meter for the floor has been evaluates as $P_{1}=28.12 \mathrm{kN} / \mathrm{m}$ and for the cover as $P_{2}=23.52 \mathrm{kN} / \mathrm{m}$.

Regarding the input motion, seismic inputs have been applied at the conventional bedrock. They represent the scenario earthquakes expected for the given area and for a specified period of return.

So, the chosen inputs are: three synthetic accelerograms evaluated assuming the source to be along the HybleanMaltese fault and generating the 1693 seismic ground motion scenario, assumed as a level I earthquake scenario
[Grasso et al., 2005; Laurenzano et al., 2004]; three synthetic accelerograms evaluated assuming the source to be along the Hyblean-Maltese fault and generating the 1818 seismic ground motion scenario, assumed as a level II earthquake scenario [Grasso et al., 2005]; one accelerogram recorded during the 1990 earthquake at the Sortino station. In order to fit the accelerograms at the reference area, they have been scaled at the same maximum expected acceleration ( $\mathrm{PHA}=0.282 \mathrm{~g}$ ), corresponding to the SLV state (i.e. the limit state for the safety of human life) and considering the building as "strategic" type (corresponding to the return period of 975 years), according to NTC [2008]. As regards the synthetic seismograms they

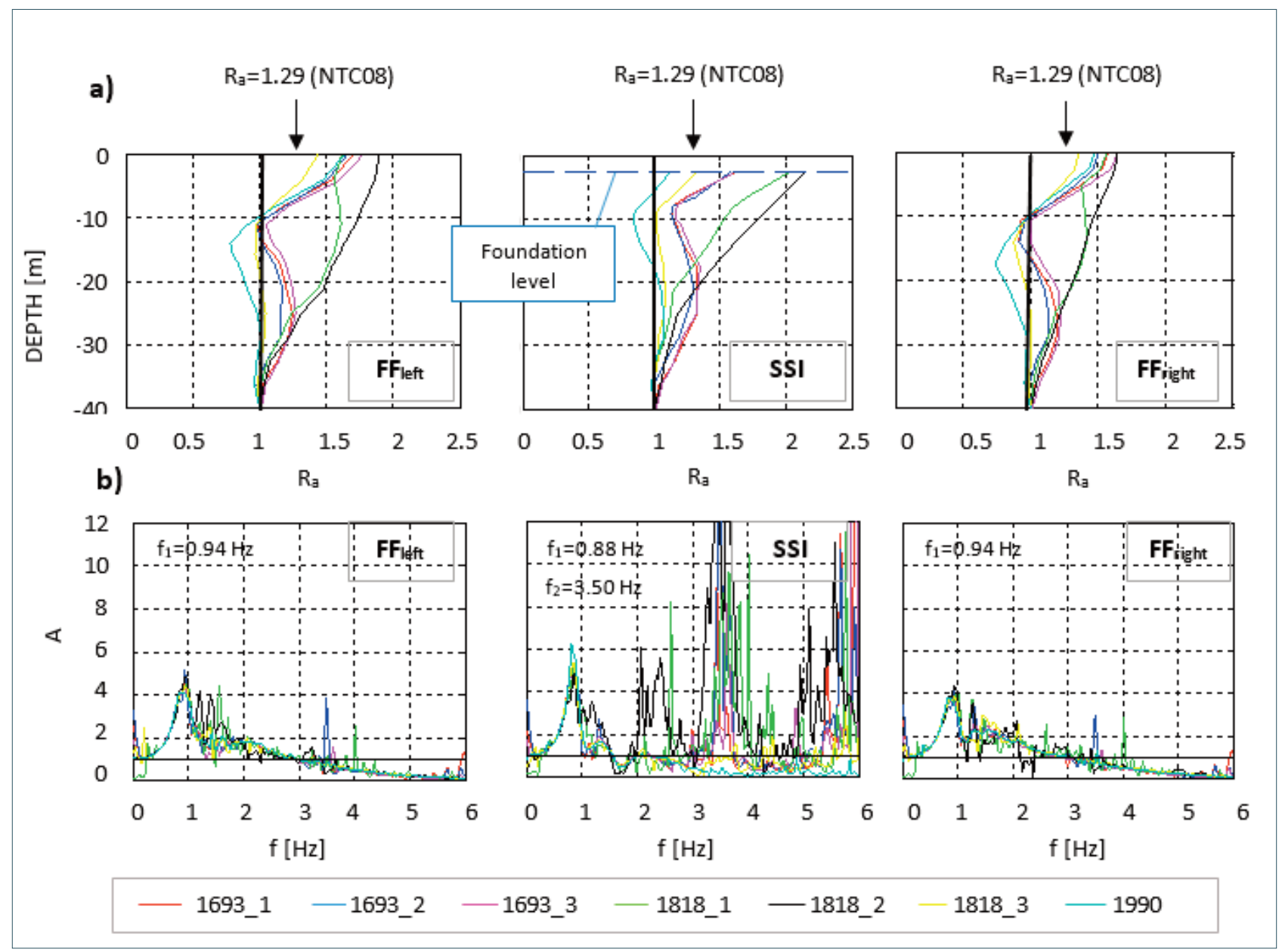

FIGURE 9. Results of 2D FEM analyses: (a) amplification ratios; (b) amplification functions. 
were scaled using attenuation relations, considering the epicentral distances for both the earthquake scenarios. Numerical simulations were performed respectively along 2-D and 3-D vertical planes containing both source and receivers, through complex geological structures; it permits to estimate the effects of deep crustal structures, superficial geology and irregular topography on the ground shaking [Grasso et al., 2009a,b, 2012; Castelli et al., 2016a,b].

Figure 7 shows the time histories and the values of the predominant frequencies and Arias intensities.

\section{MAIN RESULTS IN THE TIME AND IN THE FREQUENCY DOMAIN}

\subsection{RESULTS ACHIEVED WITH G AND D EVALUATED ACCORDING TO EC8}

\subsubsection{RESULTS BY 2D FEM IN TERMS OF AMPLI- FICATION RATIOS AND AMPLITUDE FUNC- TIONS}

Regarding the three different alignments shown in Figure 8, the results of the first 2D FEM analyses are presented in terms of amplification ratio $R_{a}$ (that is the ratio between the maximum acceleration at a fixed depth and the maximum acceleration at the base of the soil deposit; Figure 9a) and amplification function $A$ (that is the ratio between the Fourier spectrum at a fixed depth and the Fourier spectrum at the base of the soil deposit; Figure 9b): two on the left and on the right of the structure, i.e. in free-field conditions (named $\mathrm{FF}_{\text {left }}$ and $\mathrm{FF}_{\text {right }}$, respectively) and one under the structure (named SSI). Figure 9a shows no significant amplification from $40 \mathrm{~m}$ up to $35 \mathrm{~m}$, for all the three alignments. Then, in the $\mathrm{FF}_{\text {left }}$ and $\mathrm{FF}_{\text {right }}$ alignments quite all the inputs de-amplify from $35 \mathrm{~m}$ to $10 \mathrm{~m}$ and suffer a strong amplification in the last $10 \mathrm{~m}$ up to ground level. At the $\mathrm{FF}_{\text {left }}$ alignment there are values greater than those achieved for the $\mathrm{FF}_{\text {right }}$ alignment (about 10\%); this result is due to the major thickness of the first two layers "sand" and "clay1" and to the absence of the "clay2" layer, that has better mechanical characteristics than the first two ones, along the $\mathrm{FF}_{\text {left }}$ alignment. In the SSI alignment, the trends of $R_{a}$ are similar to those obtained in free-field conditions, but the existence of the struc-

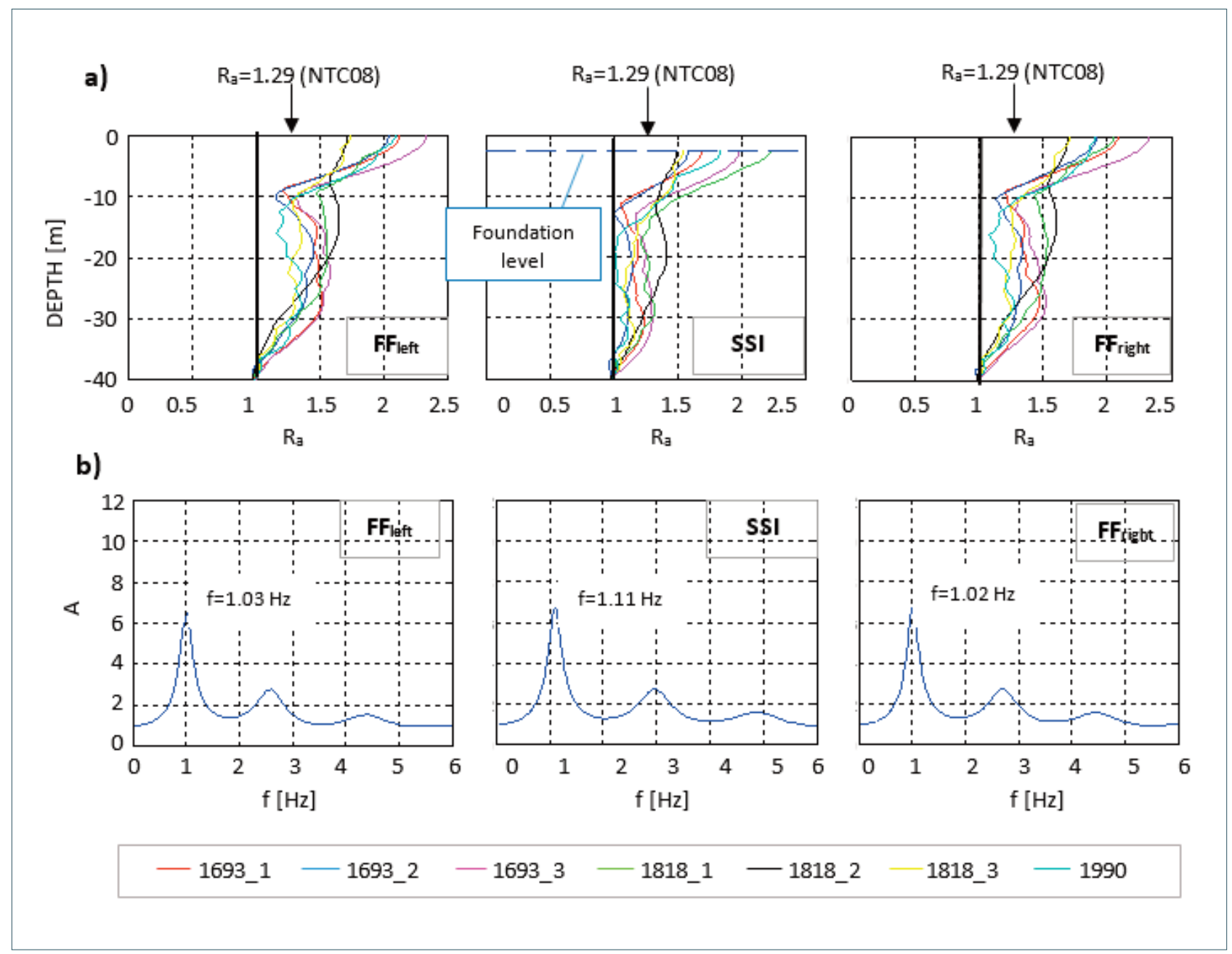

FIGURE 10. Results of 1D analyses: (a) Amplification ratios; (b) amplification functions. 
ture increases the amplification of quite all the signals. The computed amplification ratios are generally significantly higher than that suggested by NTC [2008], equal to 1.29. As for the comparison shown in Figure $9 \mathrm{~b}$, it is evident that, for the FF conditions, the natural frequency of the soil is $0.94 \mathrm{~Hz}$ regardless of the soil profile. Along the SSI alignment, the soil changes its frequency content: the first two fundamental frequencies of the system are $f_{1}=0.88 \mathrm{~Hz}$ and $f_{2}=3.5 \mathrm{~Hz}$.

\subsubsection{RESULTS BY 1D ANALYSIS IN TERMS OF AM- PLIFICATION RATIOS AND AMPLITUDE FUNC- TIONS}

The results of the FEM 2D analyses have been compared with simpler and widely used 1D analyses, here performed by means of STRATA numerical code [Kottke et al., 2008]. All the three alignments $\mathrm{FF}_{\text {left }}$, SSI and $\mathrm{FF}_{\text {right }}$ shown in Figure 8 have been considered while the presence of the structure is ignored. Linear-equivalent-elastic analyses have been performed, considering the soil-nonlinearity as before explained. The achieved results are once more presented in terms of amplification ratio $R_{a}$ (Figure 10a) and amplification function $A$ (Figure 10b).

By 1D analyses there are not evident differences among the three alignments, because all represents freefield conditions and, moreover, it is not possible to model the inclination of the soil layers. The natural frequency of the soil is always about $1.04 \mathrm{~Hz}$, which is similar to that obtained by the FEM-2D modelling in free-field conditions $(f=0.94 \mathrm{~Hz})$, but different to that estimated including the structure in FEM 2D analyses. This is one of the big limits of 1D modelling.

\subsubsection{COMPARISON BETWEEN 1D AND 2D ANALYSES IN TERMS OF RESPONSE SPECTRA}

Figure 11 shows the average spectra achieved by FEM-2D modelling (red line, average spectrum), by 1Dmodeling (green line, average spectrum) and according to NTC [2008] (blue line), obtained by setting a structural damping of $8 \%$.

It is possible to notice that between the average spectra achieved for the free-field conditions $\left(\mathrm{FF}_{\text {left }}\right.$ and $\mathrm{FF}_{\text {right }}$ ) there are not substantial differences. Moreover, 2D and 1D analyses show the same fundamental periods: a first main fundamental period $T=0.42 \mathrm{~s}$ and a second less important fundamental period $T=1.27 \mathrm{~s}$ can be observed both in 1D and 2D analyses. By the way, 1D analyses show higher spectral accelerations. For the central alignment (SSI alignment), the most important fundamental period is equal to $1.27 \mathrm{~s}$ considering DSSI, highlighting once more how can be erroneous consid- ering only FF conditions, i.e. neglecting DSSI.

In general, for $\mathrm{T} \approx 0.8-3 \mathrm{~s}$, the average spectra given by the $1 \mathrm{D}$ and $2 \mathrm{D}$ analyses are more conservative then that given by NTC [2008]. Along the central alignment (SSI alignment), by the 2D full-coupled soil-structure analysis the maximum spectral acceleration $S_{\mathrm{e}, \max }=$ $1.03 \mathrm{~g}$ at $T=1.27 \mathrm{~s}$ is obtained, while by the $1 \mathrm{D}$ analysis $S_{\mathrm{e}, \max }=1 \mathrm{~g}$ at $T=0.42 \mathrm{~s}$ is obtained. The natural period of the structure fixed at the base is $T_{F B}=0.4 \mathrm{~s}$. The natural period of the structure including the subsoil is $\mathrm{T}_{\text {DSSI }}=1.13 \mathrm{~s}$.

Thus, according to $T_{F B} 1 \mathrm{D}$ analysis is more severe than NTC [2008], while 2D analysis is less severe than NTC08. According to $T_{D S S I}$ and both $1 \mathrm{D}$ and $2 \mathrm{D}$ analysis are more severe than NTC [2008] and in any case 1D analysis is the most severe. Moreover, considering 2D full-coupled analysis moving from $T_{F B}$ to $T_{D S S I}$ the spectral acceleration decreases; moving from $T_{F B}$ to $T_{D S S I}$ the spectral acceleration decreases. Thus, a very careful evaluation of the design period and DSSI phenomena should be performed.

\subsection{RESULTS BY 2D FEM ANALYSES WITH G AND D EVALUATED ACCORDING TO THE ACHIEVED STRAIN LEVEL}

In the second phase of the FEM analyses, the values of $G$ and $D$ have been chosen according to the $G / G_{0}$ vs $\gamma$ and $D$ vs $\gamma$ curves obtained by the performed RCT shown in Figure 6, considering the effective strain level $\gamma$ obtained for each soil layer and for each different input, according to an iterative sub-routine. In the following, for lack of space, the results of this iterative procedure are shown just for the 1613-1 seismic input (Figures 12,14).

Then, with reference to the same three alignments shown in Figure 8, the results of the 2D FEM analyses are presented in terms of amplification ratio Ra (Figures 15,18) and amplification function A (Figures 19,24). Finally, a comparison regarding the structural behavior is shown, in order to understand advantages and dis-advantages in modeling soil-nonlinearity according to the very easy-to-use suggestions by EC8 or according to more time expensive iterative procedure.

Figures 12,14 shows the results of $G / G_{0}$ and $D$ per each soil layer evaluating through the iterative procedure, evaluating also how much they differ from the values adopted according to EC8 $\left(G / G_{0}=0.36\right.$ and $D=$ $10 \%)$. In particular, Figure 12 shows the convergence results of $G / G_{0}$ and $D_{0}$ for the SSI alignment, instead the values regarding the FF alignments, as well as for the SSI alignment, are shown in Figure 13. Finally, Figure 14 shows the $G / G_{0}$ curves with the achieved strain 


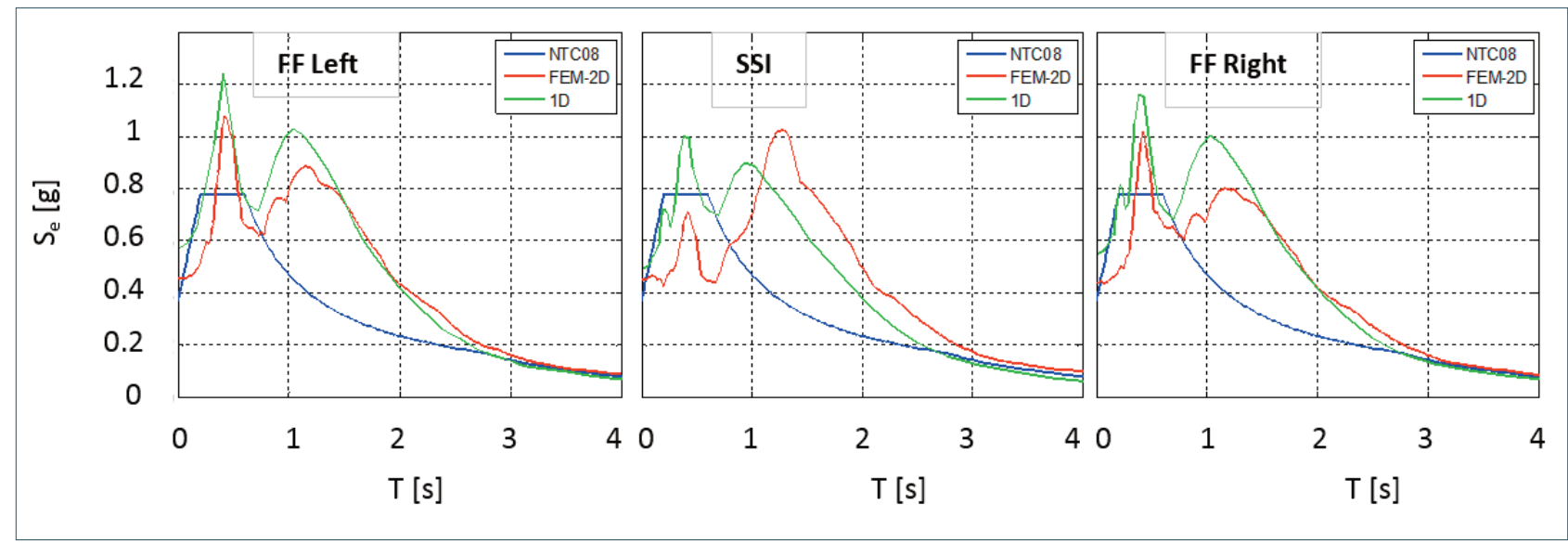

FIGURE 11. Comparison among average elastic response spectra by NTC [2008], FEM-2D analyses and 1D analyses, along the three alignments.

level per each soil layer and per the three alignments.

Regarding $G$, the values due to the iterative procedure stay in the range proposed by EC8 $( \pm 0.36)$ and the medium value is exactly 0.36 . In particular, the degradation of $G$ for the first layer is comparable to the value proposed by EC8; instead for the other layers the degradation is minor, especially for A1 and A2-1 layers. This is due to the major stiffness of clays [Crespellani, 2010]. Indeed, the last layers have also a degradation similar to the first layer, even if they are clays: this is due to the reached high strain level. Regarding the $D$ damping ratio, it increases by the depth, reaching values above $10 \%$, that is the value proposed by the EC8.

Figure 13a shows the values of G/G0 for the differ- fact for the shallow layers the presence of the structure implicates different strains in the SSI alignment in comparison to the FF alignments, and this effect decreases with depth, as shown by the curves in Figure 14. Figure $13 \mathrm{~b}$ shows the values of $D$ for the different layers and for the three investigated alignments. The achieved values are more homogeneous in respect with the values of $\mathrm{G} / \mathrm{GO}$; this is due to the fact that the soil damping is little influenced by the presence of the structure, except for the first layer S1, in which $D$ is major for the SSI alignment in respect with the FF alignment.

Finally, Figure 15 shows the $R_{a}$ profiles achieved for all the adopted inputs and for the three investigated alignments. Generally, there is a major amplification at

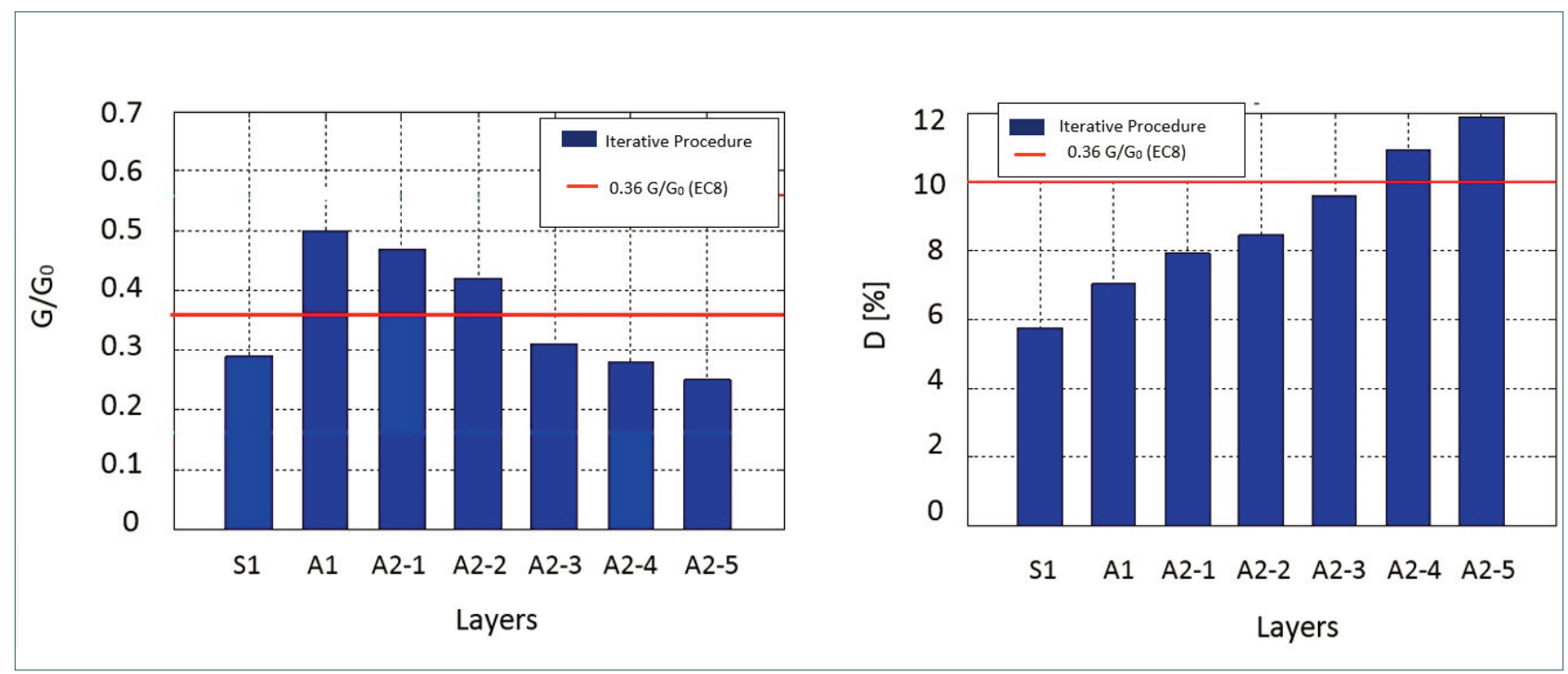

FIGURE 12. Values of $G / G_{0}$ (a) and $D$ (b) per each soil layer (1693-1 seismic input).

ent layers and for the three investigated alignments. For the deeper layers the values are similar along the three alignments; instead for the shallower layers they are different. This depends on the reached strain level, in the soil surface for the 1818 inputs, more evident for the SSI alignment. In particular, the 1818 signals amplify from the bottom to the top of the soil deposit, until to a medium $R_{a}=2.3$ at the soil surface; while the 1693 

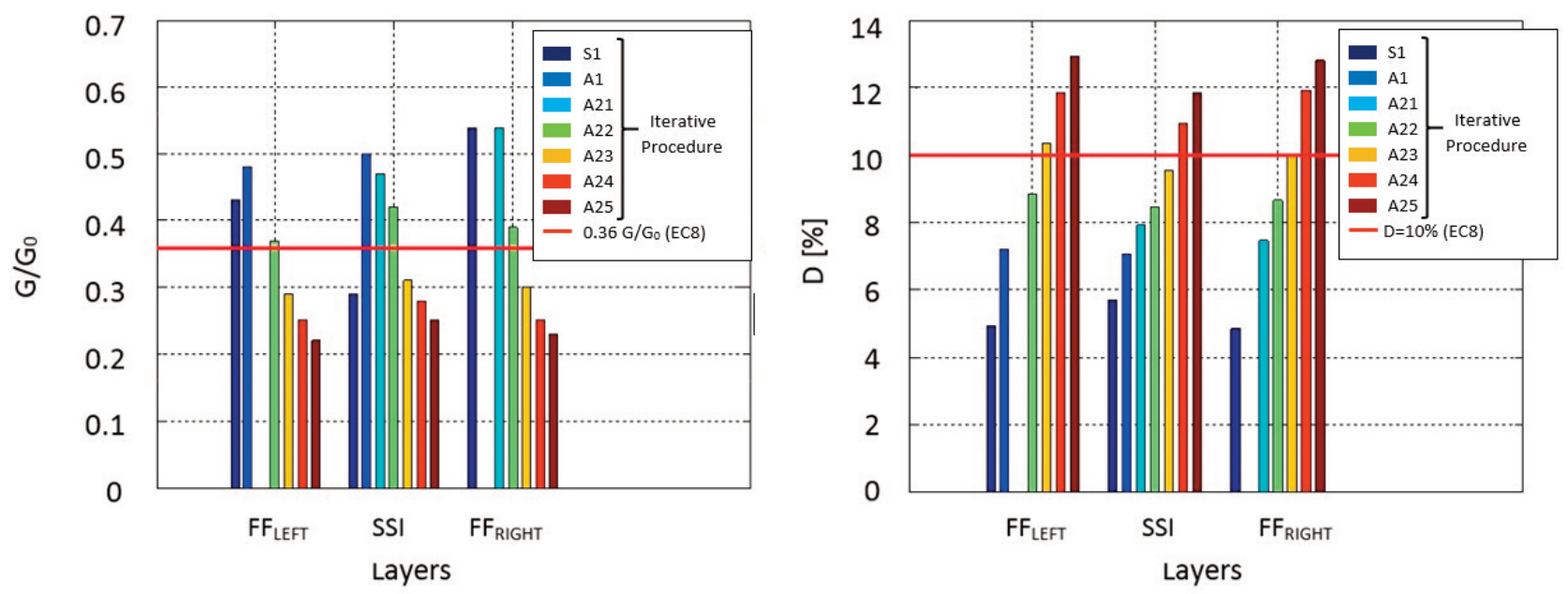

FIGURE 13. Values of $G / G_{0}$ (a) and D (b) per each soil layer and per each alignment (1693-1 seismic input).
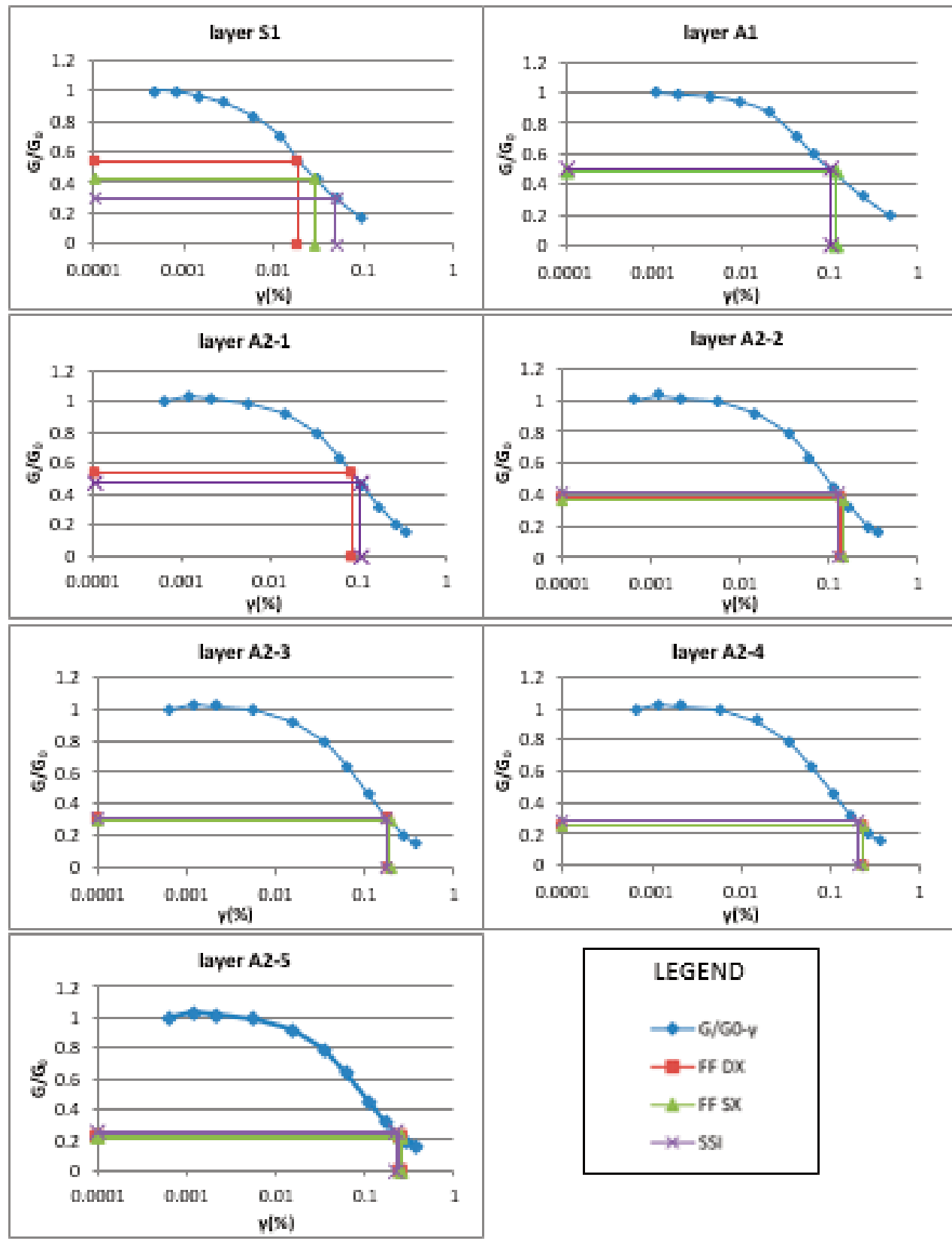

FIGURE 14. $G / G_{0}$ curves with the achieved strain level per each soil layer and per the three alignments (1693-1 input). 


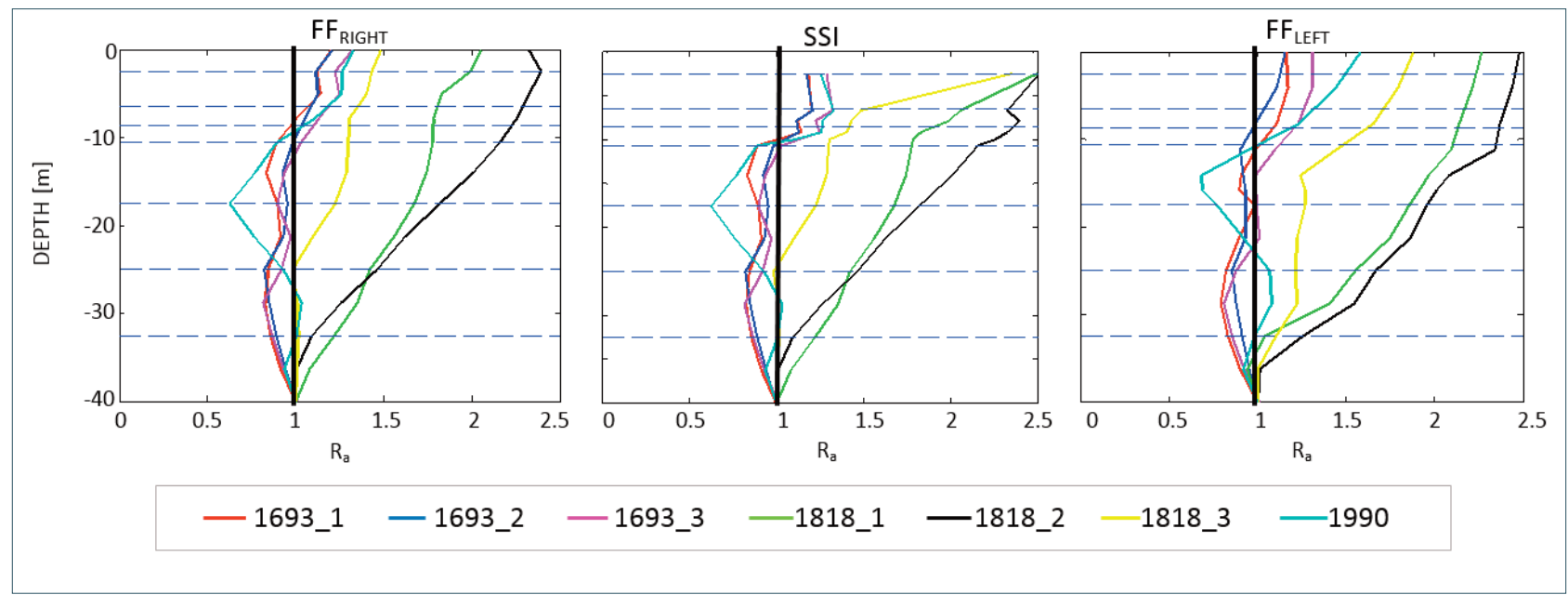

FIGURE 15. Comparison between the amplification ratios achieved by the FEM analyses based on the iterative method.

and the 1990 signals suffer a de-amplification from $40 \mathrm{~m}$ to $10 \mathrm{~m}$ and then they amplify in the shallow $10 \mathrm{~m}$, until to a medium $R_{a}=1.1$ at the soil surface, even if the second ones suffer a greater de-amplification from $40 \mathrm{~m}$ to $10 \mathrm{~m}$. The 1818 inputs produce a higher response in comparison to the 1693 and 1990 inputs, because the 1818 inputs are characterized by predominant periods very close to the natural periods of the soil, unlike the 1693 and 1990 inputs, as will be discussed in Section 4.3.1.

\subsection{COMPARISON BETWEEN THE TWO DIFFERENT 2D FEM ANALYSES REGARDING THE SOIL-NON- LINEARITY}

\subsubsection{SOIL RESPONSE}

In order to test the reliability of EC8 suggestions on soil nonlinearity, Figures 16,18 show a comparison between the amplification ratios $R_{a}$ referring to the SSI alignment for each seismic input and for the three different investigated alignments, which have been achieved by the two different ways of taking into account soil-nonlinearity in the performed 2D FEM analyses.

For the 1693 inputs (Figure 16), the values achieved by the iterative procedure are minor than the values achieved considering soil nonlinearity according to EC8; in particular, the signals suffer a de-amplification from $40 \mathrm{~m}$ to $10 \mathrm{~m}$ and then they amplify in the shallow $10 \mathrm{~m}$, until to a medium $R_{a}=1.2$ at the soil surface for the three inputs. For the 1818 inputs (Figure 17), the values achieved by the iterative procedure are much greater than the values achieved considering soil nonlinearity according to EC8; the signals suffer an amplification from the bottom to the top of the soil deposit, until to a medium $R_{a}=2.4$ at the soil surface for the three inputs. Finally, the $R_{a}$ profiles achieved for the 1990 input (Figure 18) are similar for both the adopted procedures, even if the values obtained by the iterative procedure are just greater than the others, with a $R_{a}=$ 1.25 at the soil surface.

Differences between the two FEM analyses are due to the achieved strain level and so they are due to the different adopted $G / G_{0}$ and $D$ values. Indeed, for an high strain level (on average $\gamma=0.1 \%$ ) and so for a substantial degradation of $G$ and a corresponding substantial increase of $D$, the signals suffer a minor amplification (see Figure 16); instead, for a low strain level (on average $\gamma=0.02-0.05 \%$ ) and so for a moderate degradation of $G$ and a corresponding moderate increase of $D$, the signals suffer a major amplification (see Figure 17).

This is due to the soil nonlinearity: for high energy levels, the acceleration can decrease along the soil deposit, because the high energy levels of the seismic input cause high levels of shear strain and in turn a predominant effect of the $D$ increasing in comparison to the $G$ degradation (Figure 16); instead for low energy levels of seismic input, the acceleration increases from the bottom to the soil surface, because the low energy levels of the seismic input cause low levels of shear strain and in turn a predominant effect of the $G$ degradation increasing in comparison to the $D$ (Figure 17) [Kramer, 1996]. For these analyses, being the inputs characterized by the same PHA and the analyses performed in linear-visco-elastic field, the energy level of the seismic inputs depends only by their frequencies, as will be better observed in the following results concerning the amplification function $A$.

Moreover, according to EC8 (Table 2) $D=10 \%$ for all the soil layers; while the iterative procedure for taking into account soil non-linearity produces different values of $D$ along the soil profile, thus in this second case there is a significant heterogeneity also in terms of $D$. This certainly contributes to obtaining different results. 


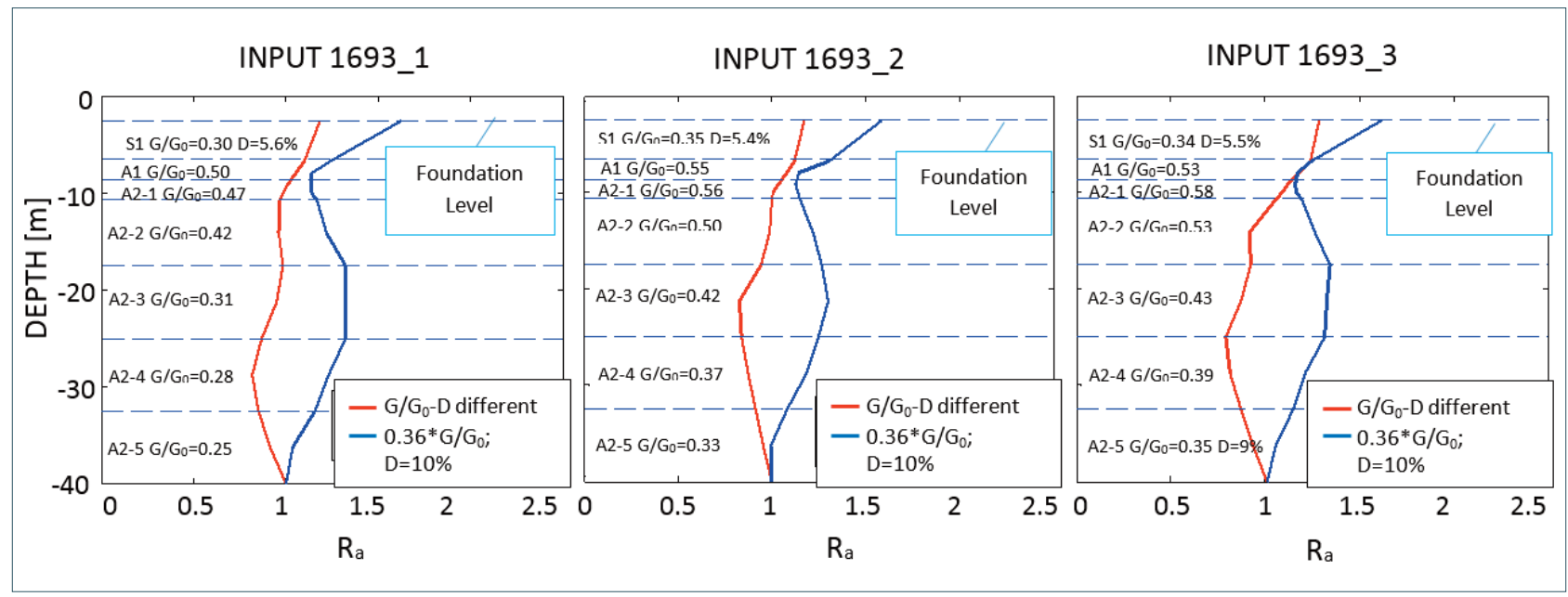

FIGURE 16. Comparison between the amplification ratios achieved by the two different ways of taking into account soil nonlinearity in 2D FEM analyses for the 1693 inputs and the SSI alignment.

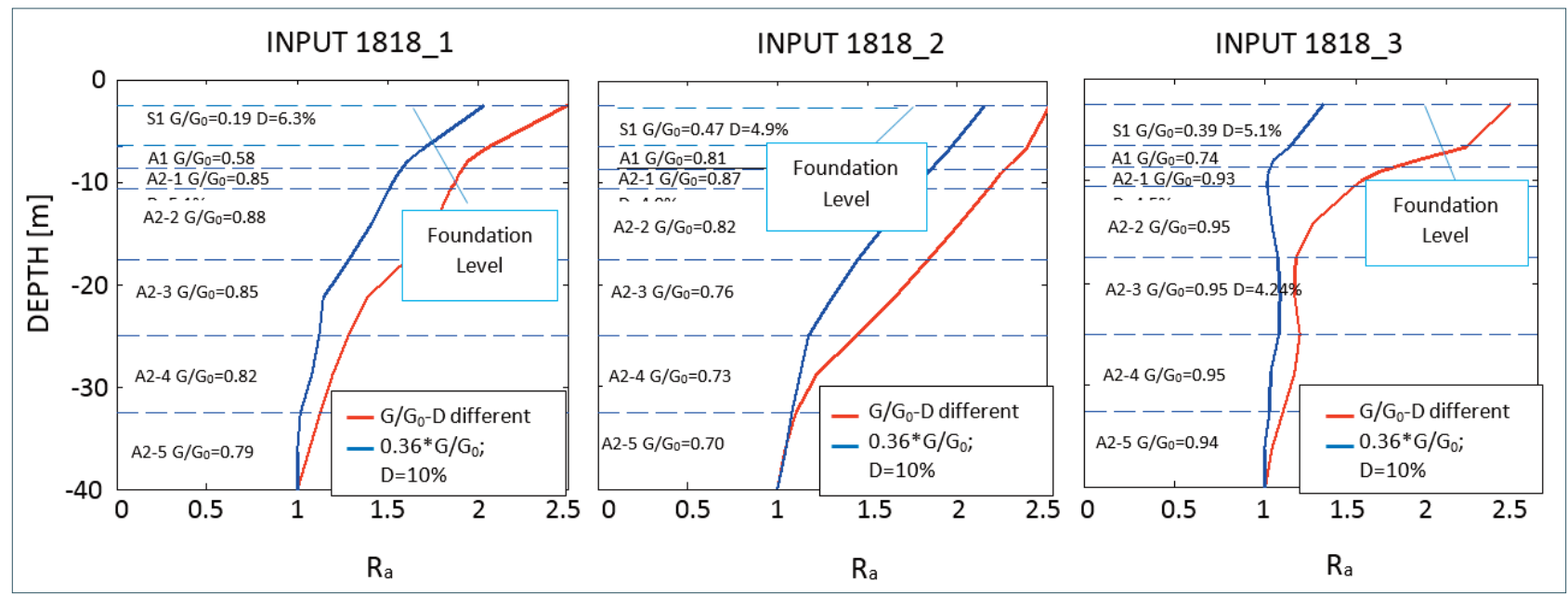

FIGURE 17. Comparison between the amplification ratios achieved by the two different ways of taking into account soil nonlinearity in 2D FEM analyses for the 1818 inputs and the SSI alignment.

Finally, the results of Figure 16,17 and 18 are certainly influenced by the ratio between the predominant periods of the inputs and the natural periods of the soil, as discussed analyzing the results of Figure 15 and as will be discussed hereafter (Figures 19,24).

The same results can be observed for the FF alignments. There is just one difference: for the SSI alignment there is a major amplification for the 1818 inputs, as it is evident in Figure 15, that shows the $R_{a}$ profiles achieved by the second FEM analyses (based on the iterative method).

Finally, Table 11 shows the values of $R_{a}$ at the soil surface for each alignment, for each seismic input and for the two different 2D FEM analyses.

Figures 19,24 show a comparison between the amplification functions $A$ achieved by the two different 2D FEM analyses, for each seismic input and for the three different investigated alignments. In particular, Figures 19,21 refer to the SSI alignment: they prove the previously discussed results about the nonlinearity. Indeed, the soil nonlinearity produces: i) a shift of the amplification peaks towards minor frequencies, that is due to the degradation of the $\mathrm{G}$ modulus; ii) a reduction of the amplification peaks, that is due to the increase of the damping ratio. This is appreciable in Figure 19: from red line (regarding the analysis according to EC8) to blue line (regarding the analysis according to the iterative procedure), the nonlinearity increases, and so its effects are more evident. For this case, the iterative procedure leads to a significant degradation of $G$ and a significant increasing of $D$. In the other Figures (Figures. $20,21)$ there is a little shift of the frequencies, but there is not a reduction of the peaks, which in same cases are greater when $G$ and $D$ are evaluated according to the iterative procedure. This is due to the different values of the damping ratio: $D$ is about $5 \%$ according to the iterative procedure, instead it is equal to $10 \%$ for the analysis according to EC8.

The results shown in Figures 19,21 depend on the achieved strain levels: the 1693 inputs and the 1990 


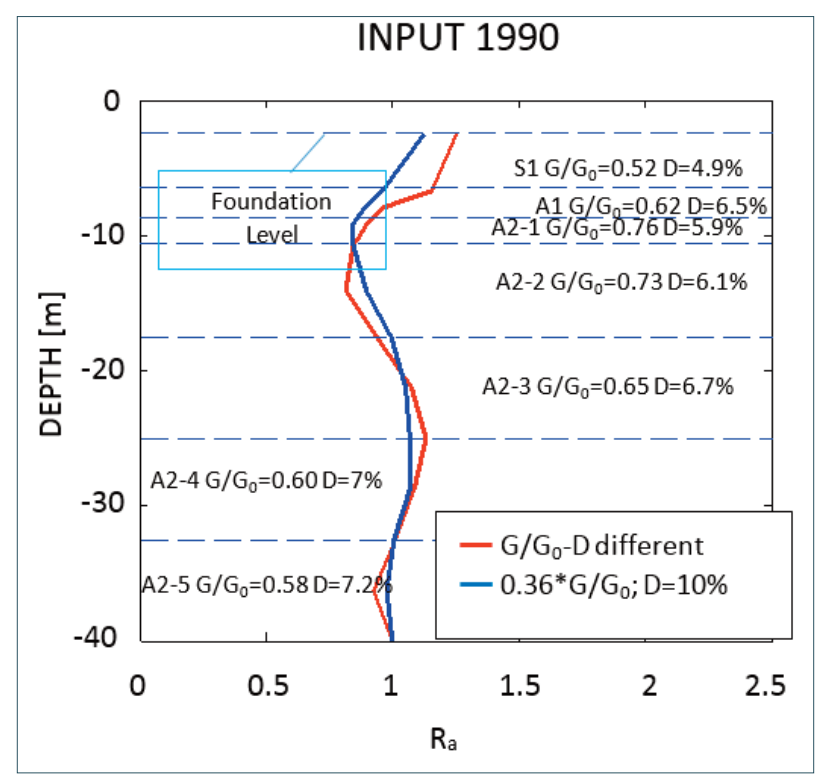

FIGURE 18. Comparison between the amplification ratios achieved by the two different ways of taking into account soil nonlinearity in 2D FEM analyses for the 1990 input and the SSI alignment.

input produce a greater strain level $(\gamma=0.1 \%)$ comparing with the strain level $(\gamma=0.02-0.05 \%)$ produced by the 1818 inputs: this implies a greater degradation of $G$ and a greater increase of $D$ due to the 1693 and 1990 cies are very close to the natural frequencies of the systems, differently from the other inputs.

Finally, Figures 22,24 show once more the comparison between the amplification functions $A$ achieved by the two different 2D FEM analyses, for each seismic input, but for the FF alignments. The two FF alignments show approximately the same predominant frequencies, as expected, due to the not great difference in the two soil profiles. Each function $A$ gives the same value of the frequency for which there is the maximum amplification both on the left and on the right. Moreover, Figures 22,24 confirm the previous considerations about the soil non-linearity achieved from Figures 19-21.

\subsubsection{STRUCTURAL RESPONSE}

Finally, the influence of the different soil modeling on the structural response is investigated. In particular, for both the FEM analyses, Figure 25 shows the shear forces $V$ at each storey, comparing them with the values achieved for the fixed-base structure $\left(V_{F X B}\right)$, with $S$ $=1.29$ proposed by the NTC [2008]. Firstly, it is important to underline that for all the investigated inputs the ratio $V / V_{F X B}$ is far from 1, thus DSSI significantly influence the structural seismic response. For the 1693 and 1990 inputs DSSI leads to the reduction of maxi-

\begin{tabular}{|c|c|c|c|c|c|c|c|c|}
\hline & INPUT & 1693-1 & $1693-2$ & 1693-3 & 1818-1 & $1818-2$ & $1818-3$ & 1990 \\
\hline \multirow{2}{*}{ FF left } & $\begin{array}{c}R_{a} \\
G / G_{O}=0.36 \\
D=10 \%\end{array}$ & 1.70 & 1.65 & 1.77 & 1.62 & 1.90 & 1.44 & 1.63 \\
\hline & $\begin{array}{c}R_{a} \\
G / G_{O}-D \text { Different }\end{array}$ & 1.17 & 1.15 & 1.31 & 2.27 & 2.68 & 1.88 & 1.58 \\
\hline \multirow{2}{*}{ SSI } & $\begin{array}{c}R_{a} \\
G / G_{O}=0.36 \\
D=10 \%\end{array}$ & 1.62 & 1.58 & 1.62 & 2.03 & 2.15 & 1.32 & 1.12 \\
\hline & $\frac{R_{a}}{G / G_{O}-D \stackrel{D}{\text { Different }}}$ & 1.18 & 1.17 & 1.28 & 2.49 & 2.50 & 2.35 & 1.25 \\
\hline \multirow{2}{*}{ FF right } & $\begin{array}{c}R_{a} \\
G / G_{O}=0.36 \\
D=10 \%\end{array}$ & 1.57 & 1.50 & 1.64 & 1.56 & 1.63 & 1.35 & 1.47 \\
\hline & $\begin{array}{c}R_{a} \\
G / G_{O}-D \stackrel{D}{\text { Different }}\end{array}$ & 1.19 & 1.20 & 1.32 & 2.05 & 2.39 & 1.48 & 1.33 \\
\hline
\end{tabular}

TABLE 11. $R_{a}$ values at soil surface.

inputs. Figures 19,21 also show the important role played by the ratios between the input predominant frequencies and the system natural frequencies. The 1818 inputs produce a more significant response in the soil (see also Figures 15,18 and 22,24) and in the structure (see Figures 25,26) because their predominant frequen- mum shear forces in comparison to the traditional design approach (fixed-base structure). For the 1818 inputs DSSI leads to an augmentation of maximum shear forces in comparison to the traditional design approach. The different inputs lead to different structural response due to their different predominant periods, 


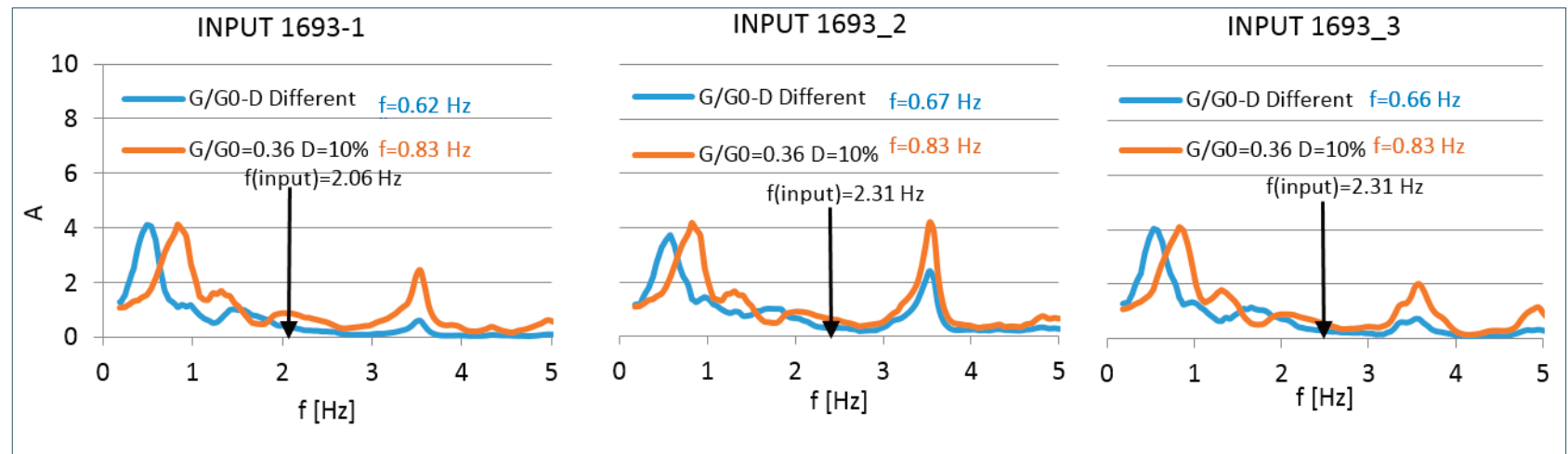

FIGURE 19. Comparison between the amplification functions achieved by the two different FEM analyses for the SSI alignment and regarding the 1693 inputs.

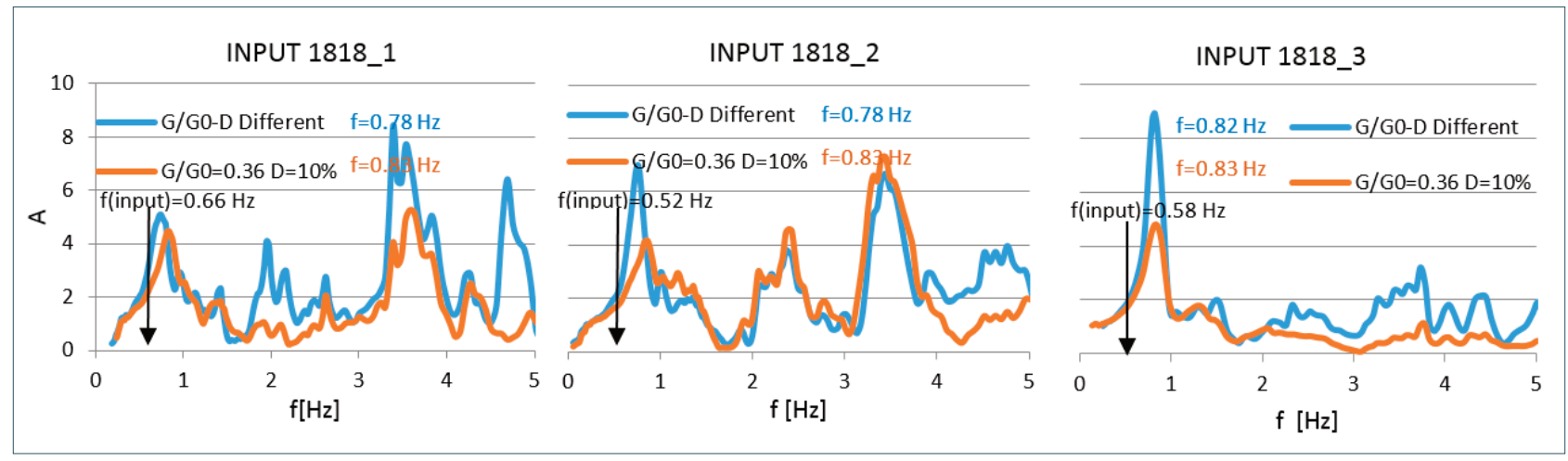

FIGURE 20. Comparison between the amplification functions achieved by the two different FEM analyses for the SSI alignment and regarding the 1818 inputs.

$T_{\text {INP }}$ : close to the natural period of the structure including the soil, $T_{\mathrm{DSSI}}$, for the 1818 inputs; far from $T_{\mathrm{DSSI}}$ for the 1693 and 1990 inputs.

Secondly, it is important to underline the different structural response in relation to the different ways used to consider soil-nonlinearity; which is appreciable above all for the 1818 inputs. In this case, the iterative procedure leads to high values of $G$ and low values of

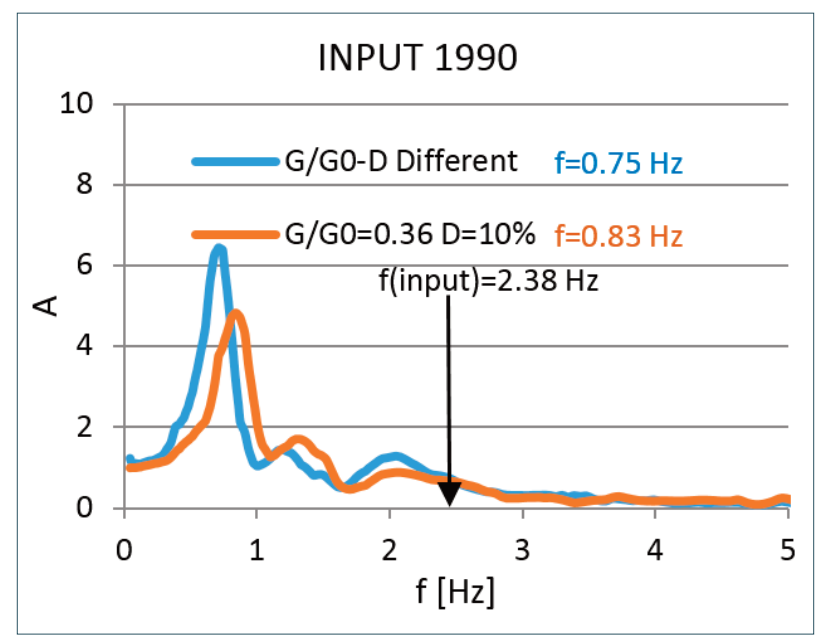

FIGURE 21. Comparison between the amplification functions achieved by the two different FEM analyses for the SSI alignment and regarding the 1990 inputs.
$D$, moreover the iterative procedure leads to a significant heterogeneity in the soil in terms of $D$ vs $z$, unlike EC8, which suggests a single value of $D$ equal to $10 \%$ for the case under examination. These reasons lead to a higher amplification ratio (Figure 17) and in turn to a greater response of the structure. So, not only in terms of soil response but also in terms of structural response it is very important to devote great attention to the estimation of soil properties and of soil nonlinearity.

Finally, peak ground acceleration at the foundation $a_{S S I}$ is compared with the peak ground acceleration at the free-field $a_{F F}$ along the FF-left alignment (Figure 26), whose soil profile is very similar to that of the SSI alignment. Deviation from the 1:1 line (orange continuous line) suggests modification of the effective foundation motion (EFM) from the free-field motion (FFM) due to DSSI.

For low accelerations the response in free-field condition is more severe than that including DSSI (aSSI < $\mathrm{aFF}$; for high accelerations the response including DSSI is more severe than that in free-field condition (aSSI > $\mathrm{aFF}$ ). The increase of aSSI from aFF implies that the input for the structure will be higher due to DSSI effects. The reason for the difference between aSSI and $\mathrm{aFF}$ is related to the ratio of the predominant periods 


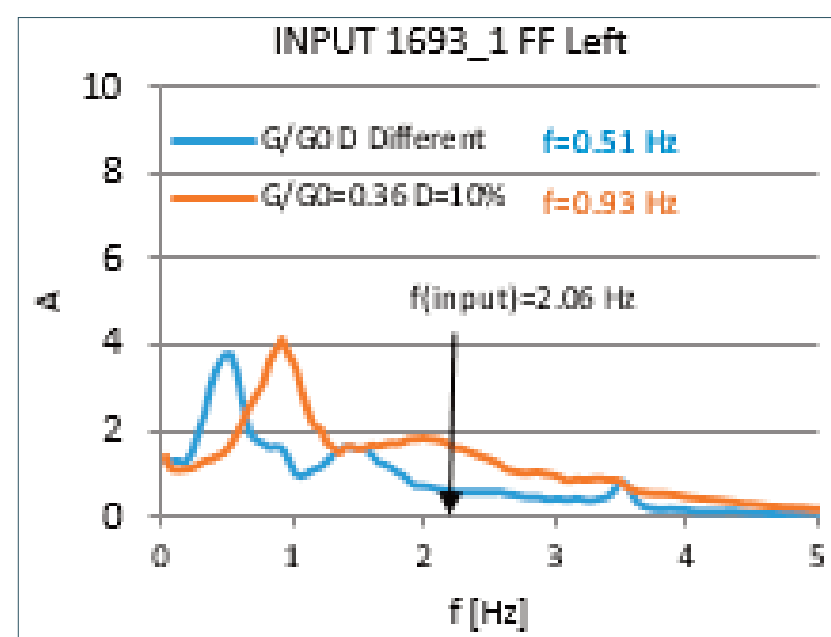

INPUT 1693_2 FF Left
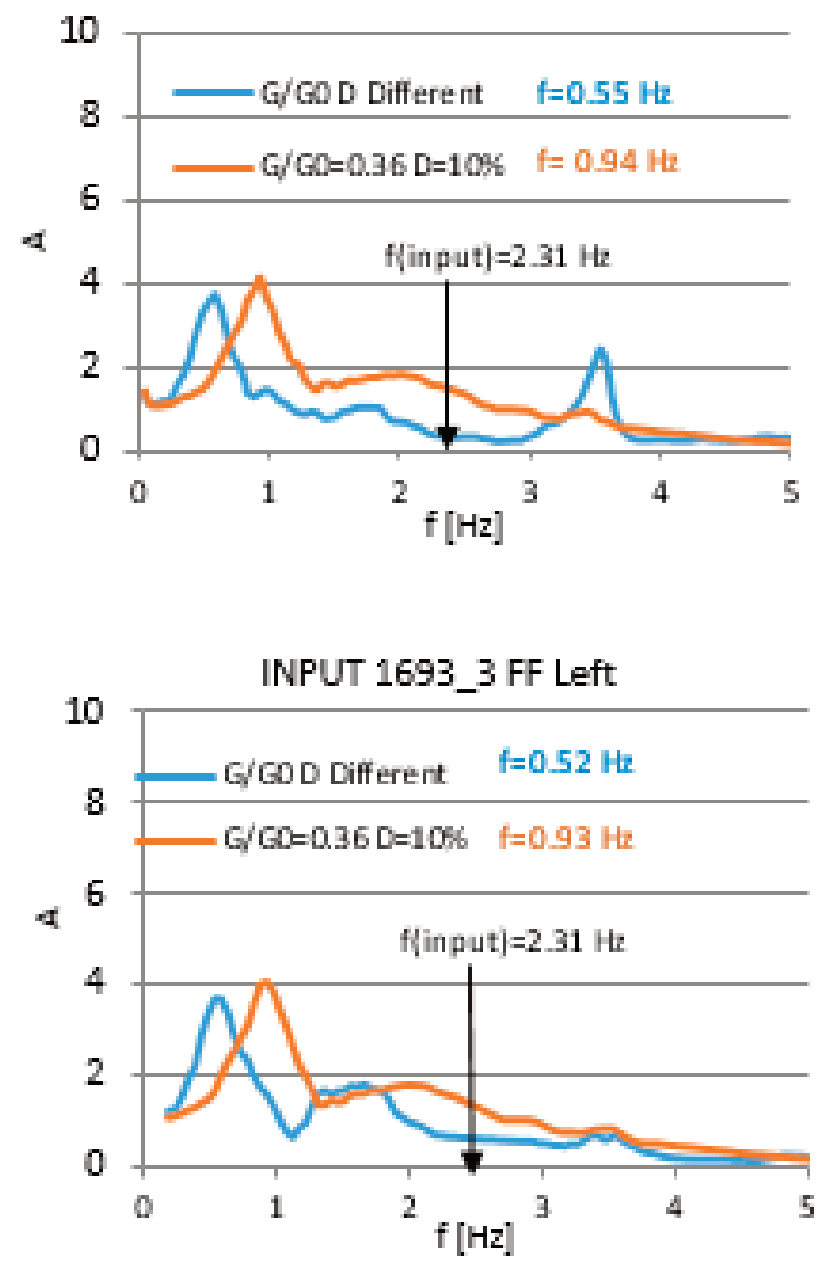

INPUT 1693_1-FF Right

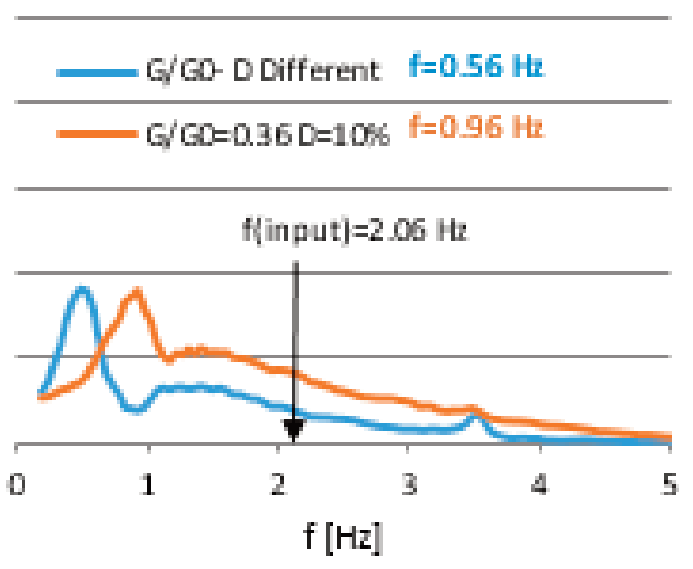

INPUT 1693_2 FF Right

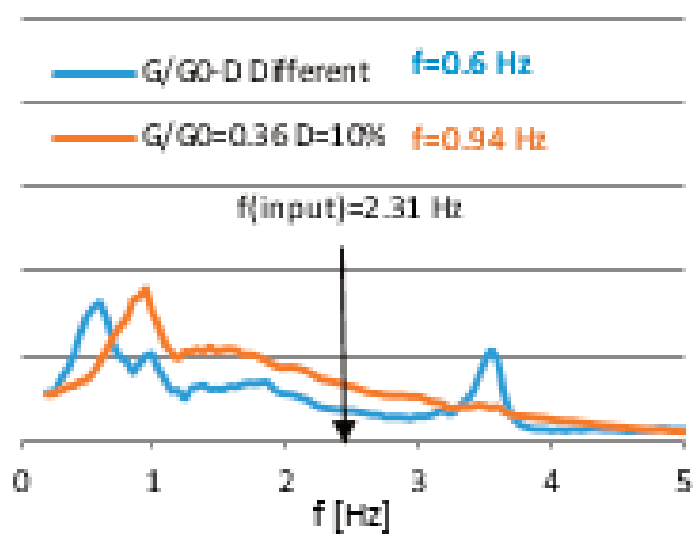

INPUT 1693_3 FF Right

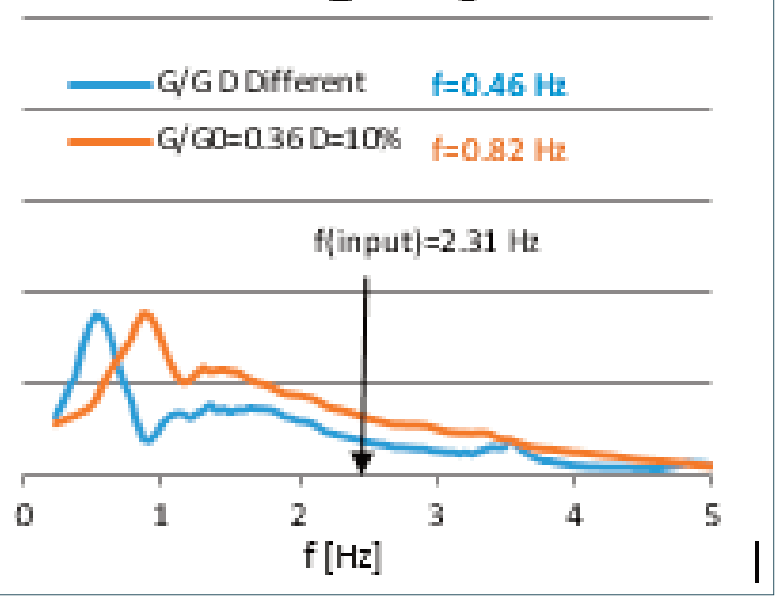

FIGURE 22. Comparison between the amplification functions achieved by the two different FEM analyses for the two FF alignments and regarding the 1693 inputs.

of the inputs TINP and the natural period of the structure including the soil, TDSSI. In most cases examined it is aSSI $<$ aFF. In two cases it is aSSI $>$ aFF, i.e. DSSI increases acceleration at the foundation. The last two cases refer to 1818_1 and 1818_3 seismic inputs. Their first predominant periods are respectively $1.51 \mathrm{~s}$ and
$1.72 \mathrm{~s}$; to which respectively TINP/TDSSI $=1.3$ and 1.5 correspond, i.e. the system is quite close to the resonant condition including DSSI $($ TDSSI $=1.13 \mathrm{~s}$ as discussed in Section 4.1.1.).

Karatzetzou et al. [2017] have recently achieved similar results. In particular, Karatzetzou et al. [2017] per- 

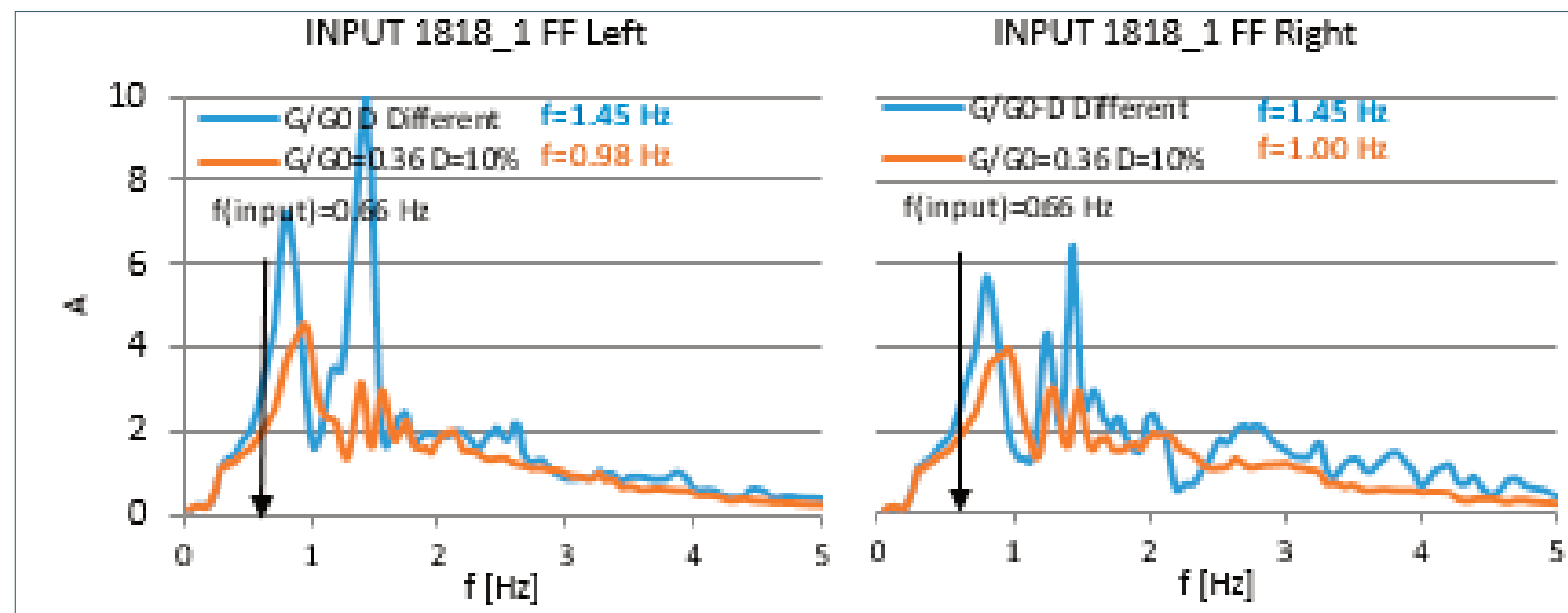

INPUT 1818_2 FF Left

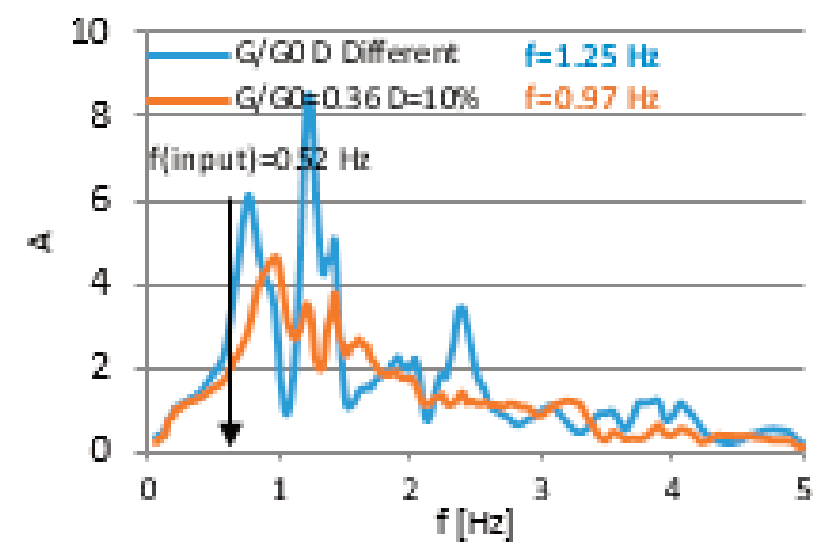

INPUT 1818_2 FF Right

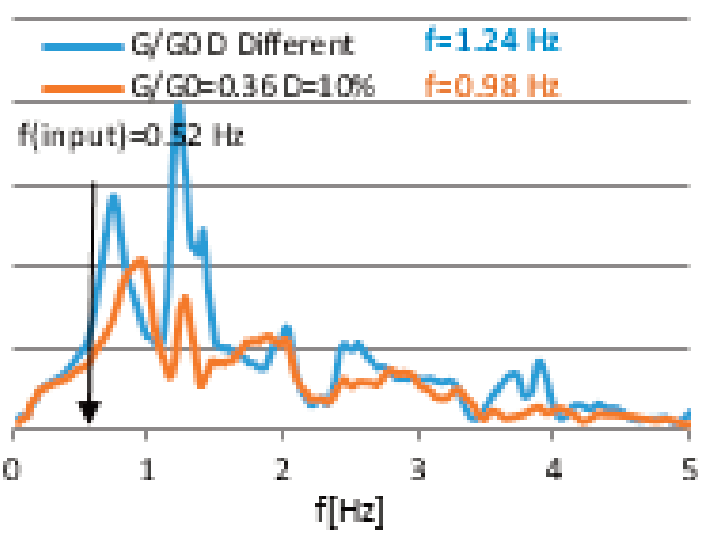

INPUT 1818_3 FF Left
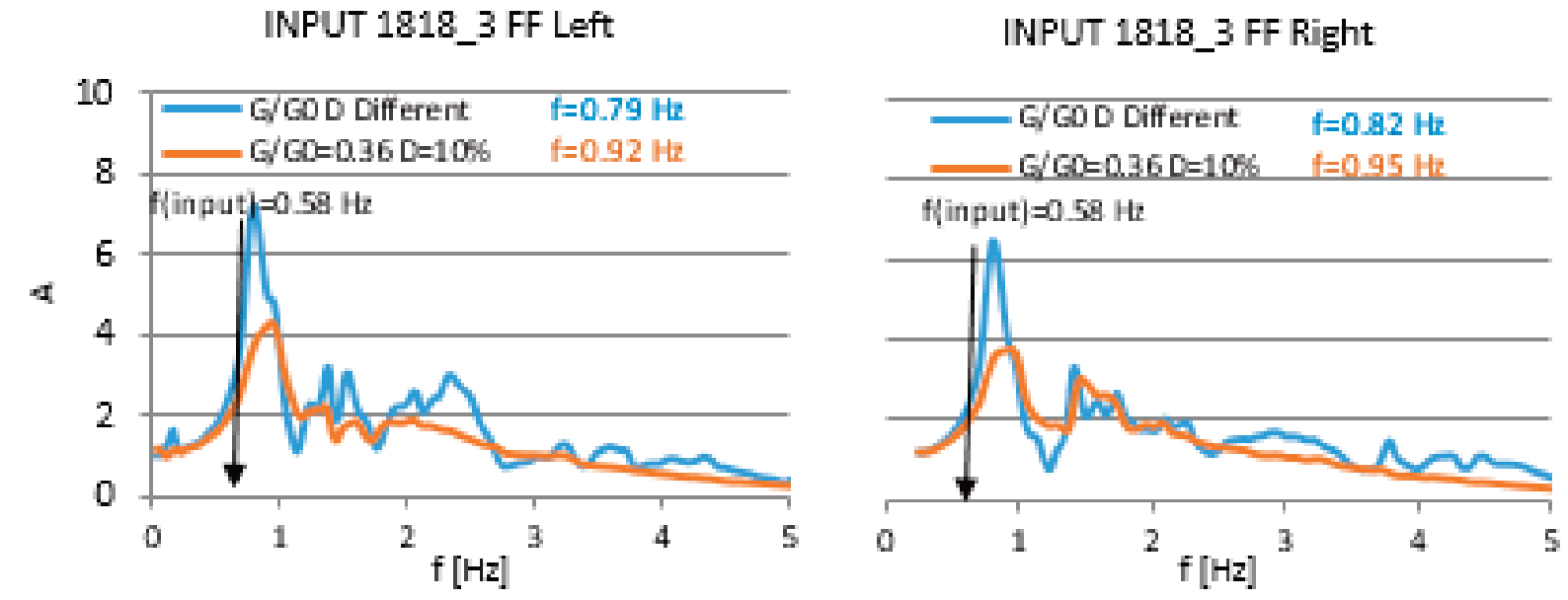

FIGURE 23. Comparison between the amplification functions achieved by the two different FEM analyses for the two FF alignments and regarding the 1818 inputs.

formed an extensive numerical parametric analysis and observed that for very short period input motions with TINP/TDSSI significantly less than 0.5 it is aSSI < aFF; in the range $0.5<$ TINP/TDSSI $<1$, an important percentage of structures revealed that aSSI < aFF, however, for an important 35\% of squatty and 10\% of more slen- der structures aSSI > aFF, this increase of aSSI from aFF in some cases might reach $150 \%$. For TINP/TDSSI $\approx 1$ it is always aSSI > aFF, As TDSSI approaches TINP, this can be viewed as progressive resonance, with DSSI increasing the maximum acceleration input to the structure. Most importantly, for $1<\mathrm{TINP} / \mathrm{TDSSI}<2$ in more 


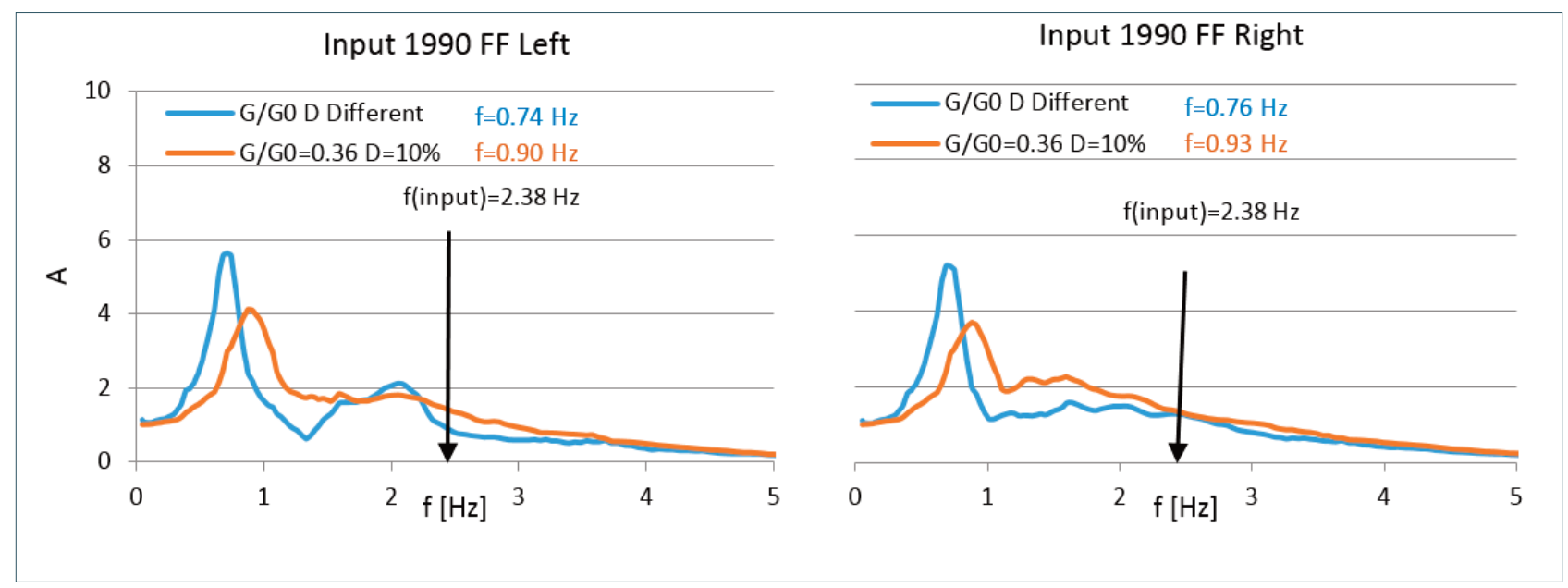

FIGURE 24. Comparison between the amplification functions achieved by the two different FEM analyses for the two FF alignments and regarding the 1990 inputs.

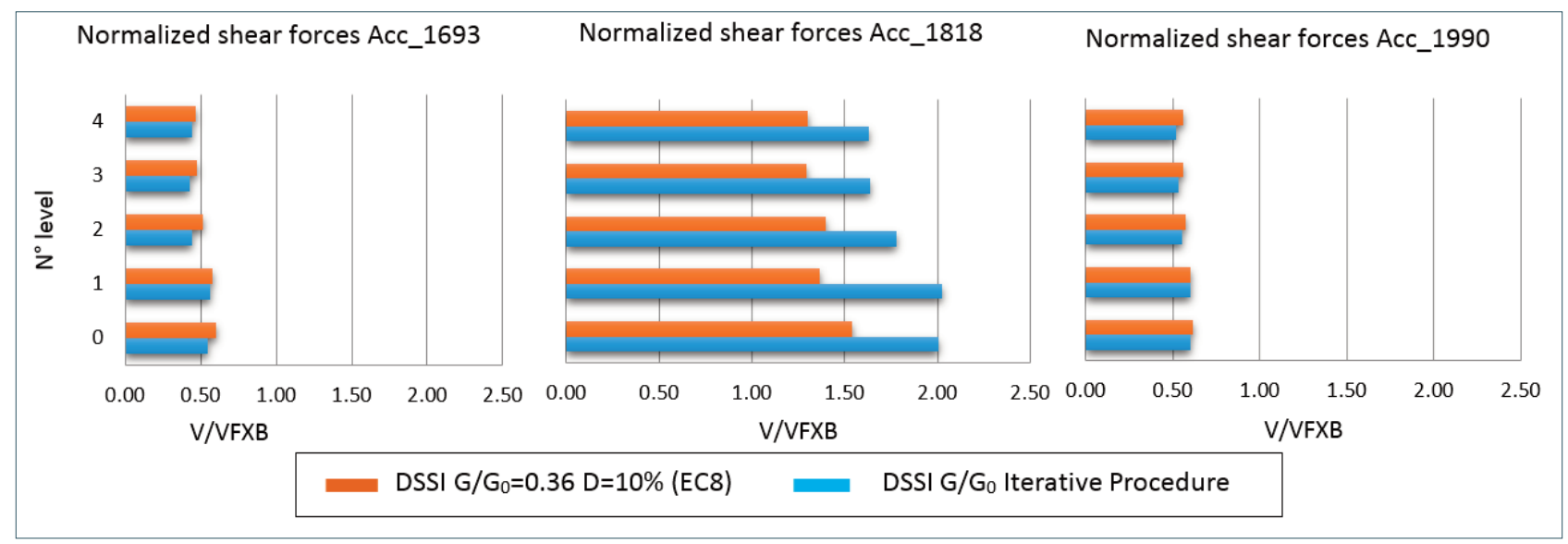

FIGURE 25. Comparison between the normalized shear forces achieved by the two different FEM analyses and for each seismic input group.

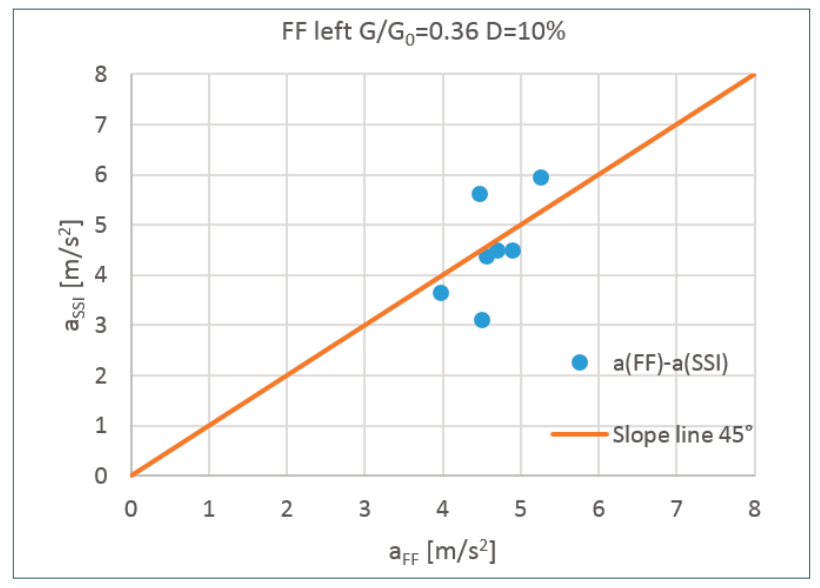

FIGURE 26. Deviation of the peak acceleration at the foundation $\left(a_{\text {SSI }}\right)$ from the peak acceleration at the freefield $\left(a_{\mathrm{FF}}\right)$.

than $95 \%$ of the cases it is aSSI $>$ aFF. For TINP/TDSSI $>2$ it is $\mathrm{aSSI} \approx \mathrm{aFF}$. Thus, the key parameter to be checked is TDSSI.

For low accelerations the response in free-field con- dition is more severe than that including DSSI $\left(a_{\text {SSI }}<\right.$ $a_{\mathrm{FF}}$ ); for high accelerations the response including DSSI is more severe than that in free-field condition $\left(\mathrm{a} a_{\mathrm{SSI}}<\right.$ $\left.a_{\mathrm{FF}}\right)$. The increase of $a_{\mathrm{SSI}}$ from $a_{\mathrm{FF}}$ implies that the input for the structure will be higher due to DSSI effects. The reason for the difference between $a_{\mathrm{SSI}}$ and $a_{\mathrm{FF}}$ is related to the ratio of the predominant periods of the inputs TINP and the natural period of the structure including the soil, $T_{\mathrm{DSSI}}$. In most cases examined it is $a_{\mathrm{SSI}}<a_{\mathrm{FF}}$. In two cases it is $a_{\mathrm{SSI}}<a_{\mathrm{FF}}$, i.e. DSSI increases acceleration at the foundation. The last two cases refer to 1818_1 and 1818_3 seismic inputs. Their first predominant periods are respectively $1.51 \mathrm{~s}$ and $1.72 \mathrm{~s}$; to which respectively $T_{\text {INP }} / T_{\text {DSSI }}=1.3$ and 1.5 correspond, i.e. the system is quite close to the resonant condition including DSSI $\left(T_{\mathrm{DSSI}}=1.13 \mathrm{~s}\right.$ as discussed in Section 4.1.1.).

Karatzetzou et al. [2017] have recently achieved similar results. In particular, Karatzetzou et al. [2017] performed an extensive numerical parametric analysis and observed that for very short period input motions with 
$T_{\text {INP }} / T_{\text {DSSI }}$ significantly less than 0.5 it is $a_{\mathrm{SSI}}<a_{\mathrm{FF}}$; in the range $0.5<T_{\mathrm{INP}} / T_{\mathrm{DSSI}}<1$, an important percentage of structures revealed that $a_{\mathrm{SSI}}<a_{\mathrm{FF}}$, however, for an important $35 \%$ of squatty and $10 \%$ of more slender structures $a_{\mathrm{SSI}}<a_{\mathrm{FF}}$, this increase of $a_{\mathrm{SSI}}$ from $a_{\mathrm{FF}}$ in some cases might reach $150 \%$. For $T_{\mathrm{INP}} / T_{\mathrm{DSSI}} \approx 1$ it is always $a_{\mathrm{SSI}}<a_{\mathrm{FF}}$, As $T_{\mathrm{DSSI}}$ approaches $T_{\mathrm{INP}}$, this can be viewed as progressive resonance, with DSSI increasing the maximum acceleration input to the structure. Most importantly, for $1<T_{\mathrm{INP}} / T_{\mathrm{DSSI}}<2$ in more than $95 \%$ of the cases it is $a_{\mathrm{SSI}}<a_{\mathrm{FF}}$. For $T_{\mathrm{INP}} / T_{\mathrm{DSSI}}>2$ it is $a_{\mathrm{SSI}} \approx a_{\mathrm{FF}}$. Thus, the key parameter to be checked is $T_{\mathrm{DSSI}}$.

\section{CONCLUSIONS}

The present paper deals with 2D FEM full-coupled soilstructure analyses for the strategic INGV (National Institute of Geophysics and Volcanology) building in Catania (Italy). Seven seismic inputs have been applied to the conventional bedrock. They represent the scenario earthquakes expected for the given area and for a specified period of return. In order to fit the accelerograms to the reference area, they have been scaled to the same maximum expected acceleration ( $\mathrm{PHA}=0.282 \mathrm{~g}$ ), corresponding to the SLV state (i.e. the limit state for the "strategic" type (corresponding to the return period of 975 years), according to NTC [2008].

Simpler 1D free-field response analyses have been also performed. Moreover, the FEM analyses have been performed according to two different approaches in order to take into account the soil non-linearity, adopting degraded shear modula and increased soil damping ratios for all the involved soil layers. In particular, in a first phase, soil non-linearity has been modeled basing on the EC8 suggestions (so, $G / G_{0}=0.36$ and $D=10 \%$ have been fixed, according to the expected peak ground acceleration at the soil surface). In a second phase, soil non-linearity has been modeled choosing the values of $G$ and $D$ according to the effective strain level obtained for each soil layer and for each different input, by means of an iterative sub-routine. As for the structure, a linear viscoelastic constitutive model has been chosen in order to focus attention on the effects of soil-nonlinearity, very often neglected in the dynamic analyses of full-coupled soil-structure systems. Nevertheless, for the masonry, the value of $E$ has been reduced to take into account the cracking of the sections, according to NTC [2008] and Ministerial Circular No. 617 of 2th February 2009.

The comparisons of the achieved results, by the 1D analyses without the structure and by the 2D FEM fullcoupled soil-structure, show that the computed stratigraphic amplification ratios are always greater than the value provided by NTC [2008]. This result is more noticeable towards the southwest side of the investigated system, because on the southwest side there is the presence of a soil layer with poorer mechanical properties. The responses in frequency domain highlight the importance of performing numerical analyses that take into account DSSI phenomena, in order to observe the changes due to DSSI not only in terms of peak acceleration but also in terms of predominant frequencies. The 1D analysis gives average response spectra more severe than that of NTC [2008] for all the significant periods and more severe than that given by the FEM-2D analysis for periods less than $1.2 \mathrm{~s}$. The DSSI analyses provide lower values of spectral acceleration for period less than $0.7 \mathrm{sec}$, but it presents much larger values for high periods, typical of structures without unrealistic fixed-base. Thus, the design periods due to DSSI phenomena should be carefully analysed through a multidisciplinary approach.

Moreover, the comparisons of the achieved results by the two different approaches for considering soil nonlinearity show the great importance of a careful evaluation for the soil effective $G$ and $D$ values. For high strain levels (on average $\gamma=0.1 \%$ ) and so for a substantial degradation of $G$ and a corresponding substantial increase of $D$, the signals suffer a minor amplification. In this situation the effect of great values of $D$ prevail on the effect of low values of $G$. Instead, for low strain levels (on average $\gamma=0.02-0.05 \%$ ) and so for a moderate degradation of $G$ and a moderate increase of $D$, the signals suffer a major amplification. In this second situation the effect of moderate values of $G$ prevail on the effect of low values of $D$. Finally, according to EC8 (Table 2) $D=10 \%$ for all the soil layers; while the iterative procedure for taking into account soil non-linearity produces different values of $D$ along the soil profile, thus in this second case there is a significant heterogeneity also in terms of $D$. This certainly contributes to obtaining different results.

As for the amplification ratio, the soil non-linearity causes a reduction of the ratio $R_{a}$, instead, as for the amplification function, the soil non-linearity causes a shift of the amplification peaks towards minor frequencies and also a reduction of these peaks. Finally, soil nonlinearity influences the structural response, as it is shown by the achieved shear forces.

Dynamic response of the structure in terms of maximum shear force per floor including DSSI is very different from that ignoring DSSI. Inputs with the same amplitudes but different predominant frequencies lead to different structural responses in terms of maximum shear forces per floor, according to the proximity to 1 of the 
ratio $T_{\mathrm{INP}} / T_{\mathrm{DSS}}$, being $T_{\mathrm{INP}}$ the input predominant periods and TDSSI the natural period of the structure including the subsoil.

The effective foundation motion deviates from the commonly evaluated free-field motion. For low acceleration at the free-field the free-field motion prevails on the effective foundation motion; for high acceleration at the free-field the effective foundation motion prevails on the free-field motion. This result is once more related to the ratio $T_{\mathrm{INP}} / T_{\mathrm{DSSI}}$.

Thus, the natural period of the structure including the subsoil, $T_{\mathrm{DSSI}}$, is a fundamental key-parameter to correctly evaluate the acceleration at the foundation and the structure response, much more than the natural period of the soil in free-field conditions or the natural period of the fixed-base structure.

Acknowledgements. Financial supports provided by the research activity POR_FESR Sicilia 2007-2013 (Line 4.1.1.1) Project, funded by the European Community, allowed the authors to achieve the results reported in this paper.

\section{REFERENCES}

Abate, G., C. Caruso, M.R. Massimino and M. Maugeri (2007). Validation of a new soil constitutive model for cyclic loading by fem analysis. Solid Mechanics and its Applications, 146, 759-768, ISSN: 09250042.

Abate, G., M.R. Massimino and M. Maugeri (2015). Numerical modelling of centrifuge tests on tunnelsoil systems. Bulletin of Earthquake Engineering, 13 (7), 28 November 2015, 1927-1951.

Abate, G. and M.R. Massimino (2016). Dynamic soilstructure interaction analysis by experimental and numerical modelling. Rivista Italiana di Geotecnica, Abate, G. and M.R. Massimino (2017a). Numerical modelling of the seismic response of a tunnel-soilaboveground building system in Catania (ltaly). Bulletin of Earthquake Engineering, 15 (1), 469491.

Abate, G. and M.R. Massimino (2017b). Parametric analysis of the seismic response of coupled tunnel-soil aboveground building systems by numerical modelling. Bulletin of Earthquake Engineering, 15 (1), 443-467.

Abate, G., M. Gatto, M.R. Massimino and D. Pitilakis (2017c). Large scale soil-foundation-structure model in Greece: dynamic tests vs FEM simulation. COMPDYN 2017, 6th ECCOMAS Thematic Conference on Computational Methods in Structural Dy- namics and Earthquake Engineering. M. Papadrakakis, M. Fragiadakis (eds.). Rhodes Island, Greece, 15-17 June 2017.

ADINA (2008). Automatic Dynamic Incremental Nonlinear Analysis. Theory and Modelling Guide, ADINA RetD, Inc. Watertown, USA.

Anastasopoulos, 1., M. Loli, T. Georgarakos and V. Drosos (2013). Shaking Table Testing of Rocking-lsolated Bridge Pier on Sand. Journal of Earthquake Engineering, 17, 1-32.

Anastasopoulos 1., Gelagoti F., Spyridaki A., Sideri J. and Gazetas G. (2014). Seismic Rocking lsolation of an Asymmetric Frame on Spread Footings. J. Geotech. Geoenviron. Eng., ASCE, 2014.140:133-151.

Bienen, B., C. Gaudin and M.J. Cassidy (2007). Centrifuge tests of shallow footing behavior on sand under combined vertical-torsional loading. Int J Phys Mod Geotech, 2, 1-21.

Capilleri, P., M.R. Massimino, and M. Maugeri (2003). Evaluation of the destructive potential of the 19971998 earthquake ground motion in the town of Sellano. WIT Transactions on the Built Environment, 72(2003): 305-314.

Capilleri, P., G. Lanzo, M. Maugeri and A. Pagliaroli (2005). Site effect evaluation in Sellano (ltaly) by 1$\mathrm{D}$ and 2-D numerical analyses. WIT Transactions on the Built Environment. 81(2005): 73-82.

Capilleri, P., M.R. Massimino, E. Motta and M. Todaro (2018). Site seismic response for a strategic building in the city of Messina by two-dimensional finite element analysis. Annals of Geophysics. This Issue.

Capilleri, P., M.R. Massimino, E. Motta and M. Todaro (2018). Two-dimensional site seismic response analysis for a strategic building in Catania. Annals of Geophysics. This lssue.

Calvi, G.M., M. Cecconi and R. Paolucci (2014). Seismic displacement based design of structures: relevance of soil-structure interaction. Geotechnical, Geological and Earthquake Engineering, 28, 241-275.

Castelli, F., A. Cavallaro and S. Grasso (2016a). SDMT soil testing for the local site response analysis. 1st IMEKO TC4 International Workshop on Metrology for Geotechnics, MetroGeotechnics 2016, 143-148.

Castelli, F., A. Cavallaro, S. Grasso and V. Lentini (2016b). Seismic microzoning from synthetic ground motion earthquake scenarios parameters: The case study of the city of Catania (1taly). Soil Dynamics and Earthquake Engineering, 88 (2016), 307-327. http://dx.doi.org/10.1016/j.soildyn.2016.07.010. ISSN: 0267-7261.

Castelli, F., A. Cavallaro, A. Ferraro, G. Grasso, V. Lentini and M.R. Massimino (2018a). Dynamic characteri- 
sation of a test site in Messina (1taly). Annals of Geophysics. This lssue.

Castelli, F., A. Cavallaro, A. Ferraro, S. Grasso, V. Lentini and M.R. Massimino (2018b). Static and dynamic properties of soils in Catania (1taly). Annals of Geophysics. This lssue.

Chatterjee, P. and B. Basu (2008). Some analytical results on lateral dynamic stiffnessfor footings supported on hysteretic soil medium. Soil Dynamics and Earthquake Engineering, 28(1), 36-43.

Circolare 02/02/09 n. 617 (2009). Istruzioni per l'applicazione delle «Nuove norme tecniche per le costruzioni» di cui al decreto ministeriale 14 gennaio 2008. Gazzetta Ufficiale Repubblica Italiana, 26-02-09 (In Italian).

Combescure, D. and T. Chaudat (2000). 1cons European program seismic tests on R/C walls with uplift; CAMUS IV specimen. ICONS Project, CEA/DRN/DMT Report, SEMT/EMSI/RT/00-27/4.

Conti R., M. Morigi and G.M.V. Viggiani G.M.V. (2017). Filtering effect induced by rigid massless embedded foundations, Bull Earthquake Eng (2017) 15:1019-1035. D01 10.1007/s10518-016-9983-7.

Crespellani, T. and J. Facciorusso (2010). Dinamica dei terreni per le applicazioni sismiche, Dario Flaccavio Editore, Palermo.

EC8-Part 5 (2003). Design of structures for earthquake resistance - Part 5: Foundations, retaining structures and geotechnical aspects. ENV 1998, Europ. Com. For Standard, Brussels.

Faccioli, E., R. Paolucci and G. Vivero (2001). Investigation of seismic soil-footing interaction by large scale cyclic tests and analytical models. In: Special presentation lecture SPL-05, Proc. of the 4th lnt Conf on Recent Advances in Geotech Earthq Eng and Soil Dyn. San Diego, USA, March 26-31, 2001.

Ferraro, A., S. Grasso, M. Maugeri and F. Totani (2016). Seismic Response Analysis in the Southern Part of the Historic Centre of the City of L'Aquila (Italy). Soil Dynamics and Earthquake Engineering. Vol. 88 (2016), 256-264. http://dx.doi.org/10.1016/j.soildyn.2016.06.009. 1SSN: 0267-7261.

Ferraro, A., S. Grasso and M.R. Massiino (2018). Site effects evaluation in Catania (ltaly) by means of 1-D numerical analysis. Annals of Geophysics. This Issue.

Gajan, S., J.D. Phalen, B.L.Kutter, T.C. Hutchinson and G. Martin (2005). Centrifuge modelling of load deformation behavior of rocking shallow foundations. Soil Dynamic and Eartquake Engineering, 25(7-10), 773-83.

Gajan, S. and B.L. Kutter (2008). Capacity, settlement and energy dissipation of shallow footings subjected to rocking. Journal of Geotechnical and Geoenvinromental Engineering - ASCE, 134(8), 1129-41.

Gazetas G. (1983). Analysis of machine foundation vibrations: State of the art. Soil Dynamic and Eartquake Engineering, 2(1), 2-42.

Gazetas, G. (1991). Foundation vibrations" in Foundation Engineering Handbook, 2nd edition, ed.

H.-Y. Fang, Chapman and Hall, New York, Chapter 15.

Gazetas G. (2015). 4th lshihara lecture: Soil-foundationstructure systems beyond conventional seismic failure thresholds. Soil Dynamics and Earthquake Engineering, 68(2015)23-39.

Gazetas, G. and M. Apostolou (2004). Nonlinear soilstructure interaction: foundation uplifting and soil yielding. In: Proc. of 3rd UJNR Workshop on SoilStructure Interaction. MenloPark, California, USA (CD-ROM).

Grasso, S., G. Laurenzano, M. Maugeri and E. Priolo (2005). Seismic response in Catania by different methodologies. Advances in Earthquake Engineering, 14, 63-79.

Grasso, S. and M. Maugeri (2009a). The Road Map for Seismic Risk Analysis in a Mediterranean City. Soil Dynamics and Earthquake Engineering, 29 (6), 1034-1045.

Grasso, S. and M. Maugeri (2009b). The Seismic Microzonation of the City of Catania (1taly) for the Maximum Expected Scenario Earthquake of January 11, 1693. Soil Dynamics and Earthquake Engineering, 29 (6), 953-962.

Grasso, S. and M. Maugeri (2012). The Seismic Microzonation of the City of Catania (Italy) for the Etna Scenario Earthquake (M=6.2) of February 20, 1818. Earthquake Spectra, 28 (2), 573-594.

Grasso S., Maugeri M. (2014). Seismic Microzonation Studies for the City of Ragusa (1taly). Soil Dynamics and Earthquake Engineering. ISSN: 0267-7261. Vol. 56 (2014), pp. 86-97. doi: 10.1016/j.soildyn.2013.10.004.

Karatzetzou, A. and D. Pitilakis (2017). Modification of Dynamic Foundation Response due to Soil-Structure Interaction, Journal of Earthquake Engineering, 4 February 2017, Pages 1-20. D01: 10.1080/13632469.2016.1264335.

Kottke, A. R. and E. M. Rathje (2008). Technical Manual for Strata. PEER Report 2008/10. Univ. of California, Berkeley, California.

Kramer S.J. (1996). Geotechnical Earthquake Enginnering. Prentice Hall, New Jersey, USA.5.

Kutter B.L. and D.L. Wilson (2006). Physical modelling of dynamic behaviour of soil-foundation-super- 
structure systems. Int J Phys Mod Geotech; 6(1), 112.

Lanzo, G. and F. Silvestri (1999). Risposta sismica locale: teorie ed esperienze. Helvius Edizioni, Napoli.

Lanzo G., A. Pagliaroli and B. D’Elia (2004). Influenza della modellazione di Rayleigh dello smorzamento viscoso nelle analisi di risposta sismica locale. Xl National Conference "Seismic Engineering in 1taly" Genova, January 25-29, 2004.

Laurenzano, G., E. Priolo, P. Klinc and A. Vuan (2004). Near fault earthquake scenarios for the February 20, $1818 \mathrm{M}=6.2$ "Catanese" event. Proc. of the Fourth International Conference on Computer Simulation in Risk Analysis and Hazard Mitigation: "Risk Analysis 2004", Rhodes, September 27-29, 2004; 81-91.

Massimino, M.R. (2005). Experimental and numerical modelling of a scaled soil-structure system. Advances in Earthquake Engineering, 14, 227-241.

Massimino M.R. and G. Biondi (2015). Some experimental evidences on dynamic soil-structure interaction. COMPDYN 2015 - 5th ECCOMAS Thematic Conference on Computational Methods in Structural Dynamics and Earthquake Engineering, 2761-2774.

Martin, C.M. and G.T. Houlsby (2001). Combined loading of spudcan foundations on clay: numerical modeling. Geotechnique, 51(8), 687-99.

Maugeri M., G. Abate G. and M.R. Massimino (2012). Soil-structure interaction for seismic improvement of Noto Cathedral (1taly). Geotechnical, Geological and Earthquake Engineering, 16(2012): 217-239.

Ministerial Circular No. 617 (2009). Instructions for the application of the «New technical standards for buildings" as per NTC, 2008 (Official Journal of the Italian Republic No. 47/2009, Ordinary Supplement No. 27). Ministry of Infrastructures and Transport 2th February, 2009 (in Italian).

NTC (2008). D.M. 14/01/08 - New technical standards for buildings, Official Journal of the Italian Republic, 14th January, 2008 (In Italian).

Mylonakis, G. and G. Gazetas (2000). Seismic soil-structure interaction: beneficial or detrimental? Journal of Earthquake Engineering, 4(3), 227-301. D01: $10.1080 / 13632460009350372$.

Pandey, B.H., Liam Finn, W.D. and Ventura C.E. (2012). Modification of free-field motions by soil-foundation-structure interaction for shallow foundations. Proc. 15th World Conference on Earthquake Engineering, Lisbon, 24-28 September, paper 3575.

Pecker, A. and C.T. Chatzigogos (2010). Non linear soil structure interaction: impact on the seismic response of structures. Proc. XIV European Conf. on
Earthquake Engineering. August 2010, Ohrid, FYROM, Keynote lecture.

Pecker, A., R. Paolucci, C.T. Chatzigogos, A.A. Correia and R. Figini (2013). The role of non-linear dynamic soil-foundation interaction on the seismic response of structures. Bulletin of Earthquake Engineering. DO1 10.1007/s10518-013-9457-0.

Pitilakis K. D., Karapetrou S. T. and Fotopoulou S. D. (2013). Consideration of aging and SSI effects on seismic vulnerability assessment of RC buildings, Bull Earthquake Eng, D01 10.1007/s10518-0139575-8.

Prasad, S.K., 1. Towhata, G.P. Chandradhara and P. Nanjunaswamy (2004). Shaking table tests in earthquake geotechnical engineering. Current Science, 87(10), 1398-404.

Renzi, S., C. Madiai and G. Vannucchi (2013). A simplified empirical method for assessing seismic soilstructure interaction effects on ordinary shear-type buildings. Soil Dynamics and Earthquake Engineering, 55, 100-107.

Ueng, T.S., M.H. Wang, M.H. Chen, C.H. Chen and L.H. Peng (2006). A large biaxial shear box for shaking table test on saturated sand. Geotechnical Testing Journal, 29(1), 1-8.

Ugalde, J.A., B.L. Kutter, B. Jeremic and S. Gajan (2007). Centrifuge modelling of rocking behaviour of bridges on shallow foundations. In: Proc. of 4 th Int. Conf. Earthq. Geotech. Eng. Thessaloniki, Greece, June 25-28 (Paper No. 1484).

Veletsos, A.S. and J.W. Meek (1974). Dynamic behaviour of building-foundation systems. Earthquake Engineering And Structural Dynamics, 3, 121-38.

Voyagaki, E., 1.N. Psycharis and G. Mylonakis (2013). Rocking response and overturning criteria for free standing rigid blocks to single-lobe pulses. Soil Dynamics and Earthquake Engineering, 46, 85-95.

*CORRESPONDING AUTHOR: Glenda ABATE, Dipartimento di Ingegneria Civile e Architettura, DICAR, Università degli Studi di Catania, Catania, Italy; email: glenda.abate@gmail.com

(C) 2018 the Istituto Nazionale di Geofisica e Vulcanologia. All rights reserved. 
A

$a_{g}$

$a_{F F}$

$a_{S S I}$

$B$

$\mathrm{CH}$

$D, D(\gamma)$

$D_{0}$

$D_{\text {o, incr }}$

DH

DSSI

E

$E_{0}$

$E_{\text {degr }}$

FEM

FF

$f$

$G, G(\gamma)$

$G_{0}$

$G_{\text {degr }}$

$g$

H

$h$

$I_{A}$

INGV

$P$

PHA

$R_{a}$

$R C T$

$S$

$S_{e}$

SDMT

$S P T$

$T$

$T_{F B}$

$T_{D S S I}$

$T_{\text {INP }}$

V

$V_{F X B}$

$V_{s}$

$V_{s, \text { degr }}$

$Z$

$\alpha$

$\beta$

$\gamma$

$\rho$

$\omega$

Amplification function in the frequency domain; i.e. the ratio between the Fourier spectrum at a fixed depth and the

Fourier spectrum at the base of the soil deposit

Ground Acceleration

Peak acceleration at the free-field

Peak acceleration at the foundation

Width of the structure

Cross hole test

Damping ratio at the current shear strain

Damping ratio at small strains

Increased damping ratio

Down hole test

dynamic soil-structure interaction

Young modulus

Young modulus at small strains

Degraded Young modulus

Finite Element Method

Free field allignment

Frequency of the input

Shear modulus at the current shear strain

Shear modulus at small strains

Degraded shear modulus

Gravity Acceleration

Height of the soil deposition

Height of the structure

Intensity Arias

National Institute of Geophysics and volcanology

Weight per meter for the floor

Input peak horizontal acceleration

Amplification ratio, i.e. the ratio between the maximum acceleration at a fixed depth and the maximum acceleration at the base of the soil deposit

Resonant column test

Soil factor by NTC08

Spectral acceleration

Seismic Dilatometer Marchetti Test

Standard Penetration Test

Period

Natural period of the fixed-base structure

Natural period of the structure including the soil

Predominant period of the input motion

Shear force

Shear force for fixed-base model

Shear waves velocity

Degraded shear wave velocity

Vertical axis (depth)

Rayleigh damping factor

Rayleigh damping factor

Shear strain

Poisson ratio

Material density

Angular frequency 